\title{
SPLASH-SXDF Multi-wavelength Photometric Catalog
}

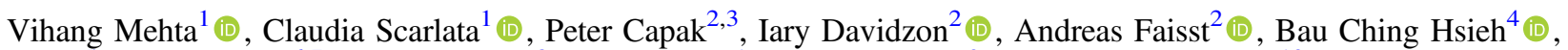 \\ Olivier Ilbert $^{5}$ (D), Matt Jarvis ${ }^{6,7}$, Clotilde Laigle ${ }^{8}$, John Phillips ${ }^{1}$, John Silverman ${ }^{9}$ (D), Michael A. Strauss ${ }^{10}$ (D), Masayuki Tanaka ${ }^{11}$ (D), \\ Rebecca Bowler $^{6}$, Jean Coupon ${ }^{12}$, Sébastien Foucaud ${ }^{13}$ (D), Shoubaneh Hemmati ${ }^{2}$ (D), Daniel Masters ${ }^{2}$ (D), Henry Joy McCracken ${ }^{14}$ (iD, \\ Bahram Mobasher $^{15}$, Masami Ouchi ${ }^{9,16}$ (D), Takatoshi Shibuya ${ }^{16}$, and Wei-Hao Wang ${ }^{4}$ (i) \\ ${ }^{1}$ Minnesota Institute for Astrophysics, University of Minnesota, 116 Church Street SE, Minneapolis, MN 55455, USA; mehta@ astro.umn.edu \\ ${ }^{2}$ IPAC, Mail Code 314-6, California Institute of Technology, 1200 East California Boulevard, Pasadena, CA 91125, USA \\ ${ }^{3}$ Cosmic Dawn Center (DAWN), Niels Bohr Institute, University of Copenhagen, Juliane Maries vej 30, DK-2100 Copenhagen, Denmark \\ ${ }^{4}$ Institute of Astronomy and Astrophysics, Academia Sinica, Taipei 10617, Taiwan \\ ${ }^{5}$ Aix Marseille Université, CNRS, LAM (Laboratoire d'Astrophysique de Marseille) UMR 7326, F-13388, Marseille, France \\ ${ }^{6}$ Oxford Astrophysics, Denys Wilkinson Building, University of Oxford, Keble Road, Oxford, OX1 3RH, UK \\ ${ }^{7}$ Department of Physics and Astronomy, University of the Western Cape, Robert Sobukwe Road, Bellville 7535, South Africa \\ ${ }^{8}$ Sub-department of Astrophysics, University of Oxford, Keble Road, Oxford OX1 3RH, UK \\ ${ }_{9}^{9}$ Kavli IPMU, Todai Institutes for Advanced Study, The University of Tokyo, Kashiwa, Chiba 277-8583, Japan \\ ${ }^{10}$ Department of Astrophysical Sciences, Princeton University, Peyton Hall, Ivy Lane, Princeton, NJ 08544, USA \\ ${ }^{11}$ National Astronomical Observatory of Japan, 2-21-1 Osawa, Mitaka, Tokyo 181-8588, Japan \\ 12 Astronomical Observatory of the University of Geneva, ch. d'Ecogia 16, CH-1290 Versoix, Switzerland \\ ${ }^{13}$ Center for Astronomy \& Astrophysics, Shanghai JiaoTong University, 800 Dongchuan Road, Shanghai 200240, People's Republic of China \\ ${ }^{14}$ Sorbonne Université, UPMC Univ Paris 06, and CNRS, UMR 7095, IAP, 98b bd Arago, F-75014, Paris, France \\ ${ }^{15}$ University of California, Riverside, 900 University Avenue, Riverside, CA 92521, USA \\ ${ }^{16}$ Institute for Cosmic Ray Research, The University of Tokyo, Kashiwa-no-ha, Kashiwa 277-8582, Japan \\ Received 2017 November 13; revised 2018 March 5; accepted 2018 March 10; published 2018 April 9
}

\begin{abstract}
We present a multi-wavelength catalog in the Subaru/XMM-Newton Deep Field (SXDF) as part of the Spitzer Large Area Survey with Hyper-Suprime-Cam (SPLASH). We include the newly acquired optical data from the Hyper-Suprime-Cam Subaru Strategic Program, accompanied by IRAC coverage from the SPLASH survey. All available optical and near-infrared data is homogenized and resampled on a common astrometric reference frame. Source detection is done using a multi-wavelength detection image including the $u$-band to recover the bluest objects. We measure multi-wavelength photometry and compute photometric redshifts as well as physical properties for $\sim 1.17$ million objects over $\sim 4.2 \mathrm{deg}^{2}$, with $\sim 800,000$ objects in the $2.4 \mathrm{deg}^{2}$ HSC-Ultra-Deep coverage. Using the available spectroscopic redshifts from various surveys over the range of $0<z<6$, we verify the performance of the photometric redshifts and we find a normalized median absolute deviation of 0.023 and outlier fraction of $3.2 \%$. The SPLASH-SXDF catalog is a valuable, publicly available resource, perfectly suited for studying galaxies in the early universe and tracing their evolution through cosmic time.
\end{abstract}

Key words: catalogs - galaxies: high-redshift - galaxies: photometry - methods: observational - techniques: photometric

\section{Introduction}

Our knowledge of galaxy formation and evolution through cosmic time has made significant headway over the past couple of decades. A large contribution to this advancement has come from large multi-wavelength photometric surveys that have made it possible to study statistically significant populations of galaxies over most of the history of the universe. The multi-wavelength coverage enables measurement of accurate photometric redshifts when combined and calibrated with well-sampled, reliable spectroscopic redshifts. Optical and near-infrared observations of galaxies over large areas have proven pivotal in tracing the evolution of the stellar mass function (e.g., Marchesini et al. 2009; Ilbert et al. 2010, 2013; Muzzin et al. 2013; Davidzon et al. 2017), the star formation mass relation (e.g., Speagle et al. 2014; Whitaker et al. 2014), and the structural evolution of galaxies (e.g., Franx et al. 2008; Bell et al. 2012; van der Wel et al. 2012; Wuyts et al. 2012; Soto et al. 2017) as well as environmental impact on galaxies (e.g., Kauffmann et al. 2004; Peng et al. 2010; Scoville et al. 2013; Darvish et al. 2016, 2017; Laigle et al. 2017).

The Subaru/XMM-Newton Deep Field (SXDF; $\alpha=02^{\mathrm{h}}$ $18^{\mathrm{m}} 00^{\mathrm{s}}, \delta=-5^{\circ} 00^{\prime} 00^{\prime \prime}$; Furusawa et al. 2008; Ueda et al. 2008 ) is one of the largest area multi-wavelength survey data sets, along with the Cosmic Evolution Survey field (COSMOS; Scoville et al. 2007). The SXDF has attracted a wealth of observational campaigns from multiple state-of-the-art groundand space-based observatories. The SXDF boasts a remarkable combination of depth ( 25-28 mag) over a large wavelength range from the optical to near-infrared and large area covered on the sky ( $\left.\gtrsim 2 \mathrm{deg}^{2}\right)$. The SXDF is perfectly suited for the study of the co-evolution of the cosmic large-scale structure, the assembly and growth of galaxies, and accurate measurement of the evolution of the global properties of galaxies through cosmic history without being significantly affected by cosmic variance.

Recently, the Spitzer Large Area Survey with HyperSuprime-Cam (SPLASH ${ }^{17}$; P. Capak et al. 2018, in preparation) program obtained additional warm-Spitzer coverage $(3.6 \mu \mathrm{m}$ and $4.5 \mu \mathrm{m})$ for the SXDF to accompany the optical coverage from the Hyper-Suprime-Cam Subaru Strategic Program (HSC-SSP; Aihara et al. 2017b), which uses the Hyper-Suprime-Cam on the Subaru $8 \mathrm{~m}$ telescope on Maunakea, Hawaii (Miyazaki et al. 2018). The combination of

17 http://splash.caltech.edu 
deep optical, near-infrared (NIR), and mid-infrared (MIR) coverage significantly improves the photometric redshifts and stellar mass estimates for high-redshift galaxies.

We generate a multi-wavelength catalog including these newly acquired data along with all available archival data on the SXDF. ${ }^{18}$ The primary goal of this paper is to homogenize and assemble all available multi-wavelength data on the SXDF on a common astrometric reference frame to measure photometry in a consistent fashion across the various bands. Furthermore, exploiting the multi-wavelength photometry, we measure the photometric redshifts and physical properties, such as stellar mass, ages, star formation rates, and dust attenuation, for all sources.

This paper is organized as follows. Section 2 describes the observations; Section 3 outlines the steps involved in homogenizing the data and assembling it on our common reference frame; the catalog creation process is detailed in Section 4; and measurements of photometric redshifts and physical properties are described in Section 5.

Throughout this paper, we use the standard cosmology with Hubble constant $H_{0}=70 \mathrm{~km} \mathrm{~s}^{-1} \mathrm{Mpc}^{-1}$, total matter density $\Omega_{m}=0.3$, and dark energy density $\Omega_{\lambda}=0.7$. All magnitudes are expressed in the AB system (Oke \& Gunn 1983).

\section{Data}

For this photometric catalog, we use the optical HSC imaging and the MIR warm-Spitzer data combined with archival optical and NIR imaging available from a variety of instruments and surveys. In this paper, we focus on a total area of $4.2 \mathrm{deg}^{2}$ centered at $(\alpha, \delta)=\left(02^{\mathrm{h}} 18^{\mathrm{m}} 00^{\mathrm{s}},-5^{\circ} 00^{\prime} 00^{\prime \prime}\right)$, matching the size of the available IRAC mosaics. In this photometric dataset, an area of $2.4 \mathrm{deg}^{2}$ has optical imaging available from HSC (hereafter, HSC-UD area). The HSC-UD area also represents the region with the deepest data and the largest wavelength coverage. Outside the HSC-UD area, there is limited coverage available in the optical from CFHTLS and MIR from Spitzer/IRAC, along with VIDEO NIR coverage on a fraction of the area.

Descriptions of the various observations included in this paper are provided below and their footprint on the SXDF is shown in Figure 1. The full list of the photometric bandpasses available from the various instruments and surveys is provided in Table 1 and the transmission curves are shown in Figure 2. Figure 3 shows the $5 \sigma$ magnitude limit maps for a selected filter from the various imaging data included in the catalog. Figure 4 shows the typical $5 \sigma$ limiting magnitude for all the photometric data included in the catalog.

\subsection{Optical and Near-infrared Data}

HSC: The Hyper-Suprime-Cam Subaru Strategic Program (HSC-SSP; Aihara et al. 2017a) covers the SXDF in the grizy filters (Miyazaki et al. 2018) using the Hyper-Suprime-Cam (Miyazaki et al. 2012) on the Subaru $8 \mathrm{~m}$ telescope on Maunakea, Hawaii. The Ultra-Deep layer of the HSC-SSP covers two fields, COSMOS and SXDF, in broadband and narrowband filters. In this paper, we include the imaging data from the HSC-SSP first public data release ${ }^{19}$ (Aihara et al. 2017b). These data go to only part of the full planned depth, as the HSC-SSP continues to collect deeper data on these fields

\footnotetext{
18 The electronic version is available for download at https://z.umn. edu/SXDF.

19 https://hsc-release.mtk.nao.ac.jp/doc/
}

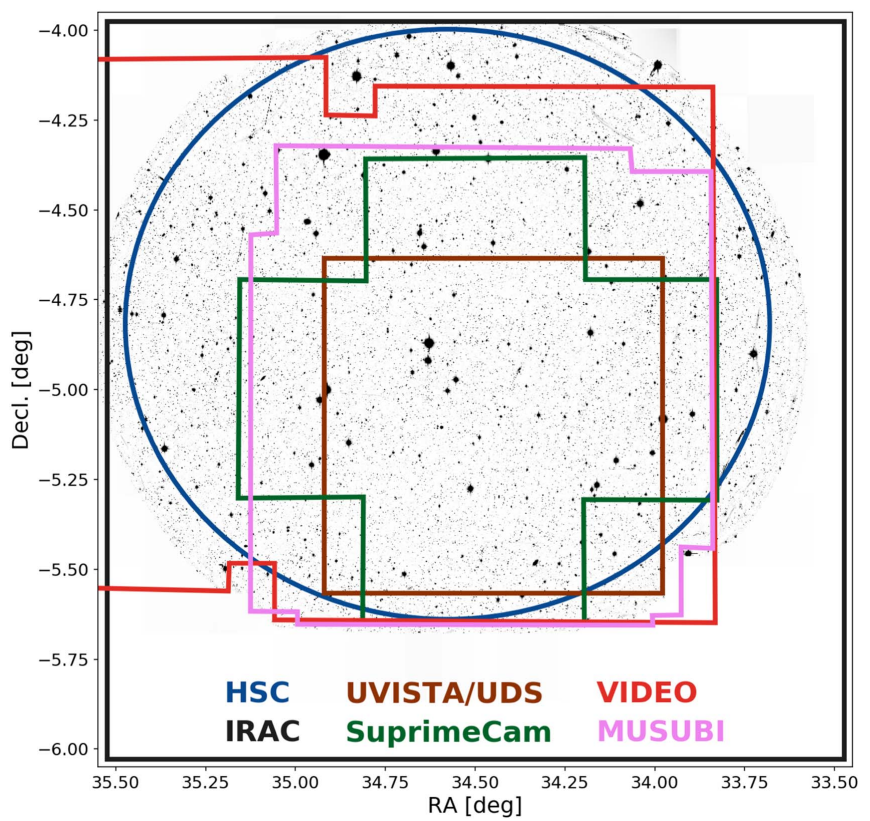

Figure 1. Coverage maps from the HSC, IRAC, and Suprime-Cam instruments, as well as the UVISTA/UDS, VIDEO, MUSUBI, and CFHTLS surveys for the SXDF shown overplotted on the HSC $y$-band mosaic. In addition to the labeled instruments/surveys, coverage from CFHTLS is available over the full mosaic.

over the coming year. The exposure times for the $g, r, i, z$, and $y$ bands are $70,70,130,130$, and 210 minutes, respectively, covering an area of $2.4 \mathrm{deg}^{2}$, with $\sim 0$ ". 6 seeing. The data are processed with hscPipe (Bosch et al. 2017). We refer the reader to Aihara et al. (2017a, 2017b) for a detailed description of the survey and data processing. The depth of the data is listed in Table 1, measured using the technique outlined in Section 4.3.2.

UDS: The Ultra Deep Survey (UDS) includes NIR imaging in the $J H K$ filters on the SXDF from the UKIRT Wide-Field Camera. We use the $J H K$ mosaics from their Data Release $11^{20}$ (DR11). The DR11 covers the UDS over the full $0.8 \mathrm{deg}^{2}$ going down to $\sim 25.3 \mathrm{mag}$ ( $5 \sigma 2^{\prime \prime}$ aperture) in the $J H K$ bands. The UDS is a part of the UKIDSS project, described in Lawrence et al. (2007). Further details on the UDS can be found in O. Almaini et al. (2018, in preparation).

VIDEO: The VISTA Deep Extragalactic Observations (VIDEO) surve ${ }^{21}$ (Jarvis et al. 2013) covers the SXDF in $Z_{Y J H K_{s}}$ filters using the VISTA Infrared Camera (VIRCAM). It reaches a $5 \sigma$ depth of $\sim 23.7$ (25.3) mag in the $K_{s}$-(Z-) band in a $2^{\prime \prime}$ aperture with a typical seeing of $\sim 0.8$ ".

Suprime-Cam: Additional optical coverage is available from the Subaru/XMM-Newton Deep Survey (SXDS; Furusawa et al. 2008), which includes $B V R_{c} i^{\prime} z^{\prime}$ filters from the Subaru Suprime-Cam covering $1.22 \mathrm{deg}^{2}$ centered at $(\alpha, \delta)=$ $\left(02^{\mathrm{h}} 18^{\mathrm{m}} 00^{\mathrm{s}},-5^{\circ} 00^{\prime} 00^{\prime \prime}\right)$ down to $\sim 27.5 \mathrm{mag}\left(5 \sigma ; 2^{\prime \prime}\right.$ aperture).

MUSUBI: The ultra-deep CFHT $u$-band stack is provided by the program Megacam Ultra-deep Survey: U-Band Imaging (MUSUBI, W.-H. Wang et al. 2018, in preparation). The MUSUBI team acquired $41.8 \mathrm{hr}$ of $u$-band integration between 2012 and 2016, using MegaCam on CFHT. The image stack

\footnotetext{
${ }^{20} \mathrm{http}: / /$ www.nottingham.ac.uk/astronomy/UDS/data/dr11.html

${ }^{21}$ http://www-astro.physics.ox.ac.uk/ video/public/Home.html
} 
Table 1

Filters Included in the Multi-wavelength Catalog

\begin{tabular}{|c|c|c|c|c|c|}
\hline $\begin{array}{l}\text { Instrument/ } \\
\text { Survey }\end{array}$ & Filters & $\begin{array}{c}\text { Central } \lambda \\
{[\mu \mathrm{m}]}\end{array}$ & $\begin{array}{c}\text { FWHM } \\
{[\mu \mathrm{m}]}\end{array}$ & $\begin{array}{c}5 \sigma \text { depth }^{\mathrm{a}} \\
{[\mathrm{mag}]} \\
\left(2^{\prime \prime} / 3^{\prime \prime}\right)\end{array}$ & $\begin{array}{r}\text { Area } \\
{\left[\mathrm{deg}^{2}\right]}\end{array}$ \\
\hline \multirow[t]{5}{*}{$\overline{\mathrm{HSC}}$} & $g$ & 0.4816 & 0.1386 & $26.84 / 26.13$ & 2.4 \\
\hline & $r$ & 0.6234 & 0.1504 & $26.36 / 25.65$ & 2.4 \\
\hline & $i$ & 0.7741 & 0.1552 & $26.11 / 25.43$ & 2.4 \\
\hline & $z$ & 0.8912 & 0.0773 & $25.52 / 24.84$ & 2.4 \\
\hline & $y$ & 0.9780 & 0.0783 & $24.79 / 24.09$ & 2.4 \\
\hline \multirow[t]{5}{*}{ SupCam } & $B$ & 0.4374 & 0.1083 & $27.40 / 26.63$ & 1.2 \\
\hline & V & 0.5448 & 0.0994 & $27.04 / 26.28$ & 1.2 \\
\hline & $R_{c}$ & 0.6509 & 0.1176 & $26.85 / 26.10$ & 1.2 \\
\hline & $i^{\prime}$ & 0.7676 & 0.1553 & $26.63 / 25.87$ & 1.2 \\
\hline & $z^{\prime}$ & 0.9195 & 0.1403 & $25.87 / 25.17$ & 1.2 \\
\hline \multirow[t]{3}{*}{$\overline{\text { UDS }}$} & $J$ & 1.2556 & 0.1581 & $25.58 / 25.09$ & 0.8 \\
\hline & $H$ & 1.6496 & 0.2893 & $25.02 / 24.56$ & 0.8 \\
\hline & K & 2.2356 & 0.3358 & $25.32 / 24.82$ & 0.8 \\
\hline \multirow[t]{5}{*}{ VIDEO } & $Z$ & 0.8779 & 0.0979 & $25.31 / 24.57$ & 1.7 \\
\hline & $Y$ & 1.0211 & 0.0927 & $24.86 / 24.15$ & 2.5 \\
\hline & $J$ & 1.2541 & 0.1725 & $24.34 / 23.74$ & 2.5 \\
\hline & $H$ & 1.6464 & 0.2917 & $24.01 / 23.38$ & 2.5 \\
\hline & $K_{s}$ & 2.1488 & 0.3091 & $23.68 / 23.07$ & 2.5 \\
\hline MUSUBI & $u$ & 0.3811 & 0.0654 & $27.38 / 26.70$ & 1.7 \\
\hline \multirow[t]{5}{*}{ CFHTLS } & $u$ & 0.3811 & 0.0654 & $25.72 / 25.20$ & $4.2^{\mathrm{b}}$ \\
\hline & $g$ & 0.4862 & 0.1434 & $26.05 / 25.50$ & $4.2^{\mathrm{b}}$ \\
\hline & $r$ & 0.6258 & 0.1219 & $25.45 / 24.88$ & $4.2^{\mathrm{b}}$ \\
\hline & $i$ & 0.7553 & 0.1571 & $24.96 / 24.33$ & $4.2^{\mathrm{b}}$ \\
\hline & $z$ & 0.8871 & 0.0935 & $23.82 / 23.06$ & $4.2^{\mathrm{b}}$ \\
\hline \multirow[t]{4}{*}{$\overline{\text { IRAC }}$} & ch.1 & 3.5573 & 0.7431 & $25.39 /-^{c}$ & 4.2 \\
\hline & $\operatorname{ch} .2$ & 4.5049 & 1.0097 & $25.13 /-^{\mathrm{c}}$ & 4.2 \\
\hline & ch.3 & 5.7386 & 1.3912 & $23.04 /-^{c}$ & 3.3 \\
\hline & ch. 4 & 7.9274 & 2.8312 & $22.90 /-^{\mathrm{c}}$ & 3.3 \\
\hline
\end{tabular}

Notes.

${ }^{\mathrm{a}} 5 \sigma$ depth in $\mathrm{AB}$ magnitudes is computed from measuring the sky variation by placing apertures in empty regions on the original images.

${ }^{b}$ This denotes the area of the CFHTLS data considered for this catalog.

${ }^{c}$ The limiting magnitudes for the IRAC channels are computed from the rms maps output from IRACLEAN by placing circular apertures in areas with no sources.

also includes $18.7 \mathrm{hr}$ of archived MegaCam $u$-band data within the SXDF. The final reduced map covers an area of $1.7 \mathrm{deg}^{2}$. The central region that receives the full $60.5 \mathrm{hr}$ of integration has an area of $0.64 \mathrm{deg}^{2}$. The central region reaches a $5 \sigma$ depth of $27.37 \mathrm{mag}$ in a $2^{\prime \prime}$ aperture.

CFHTLS: The CFHT Legacy Survey (CFHTLS) also covers the SXDF in ugriz filters using MegaCam on CFHT, as part of its wide-field coverage. We use the stacked mosaics released in the Terapix CFHTLS T0007 release. ${ }^{22}$ The median exposure times for the CFHTLS $u, g, r, i$, and $z$ bands are 250, 208, 133, 500, and 360 minutes, respectively. The seeing for these data varies for individual pointings, ranging from 0 "! $84 \pm 0$ ". 11,0 " $77 \pm 0$ ". 10 ,

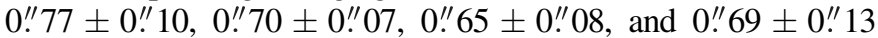
for the $u, g, r, i$, and $z$ bands, respectively. The CFHTLS coverage is available over a much larger area, thus we only consider a $\sim 2 \times 2 \mathrm{deg}^{2}$ area centered at $(\alpha, \delta)=\left(02^{\mathrm{h}} 18^{\mathrm{m}} 00^{\mathrm{s}},-5^{\circ} 00^{\prime} 00^{\prime \prime}\right)$.

\footnotetext{
${ }^{22}$ http://www.cfht.hawaii.edu/Science/CFHTLS/
}
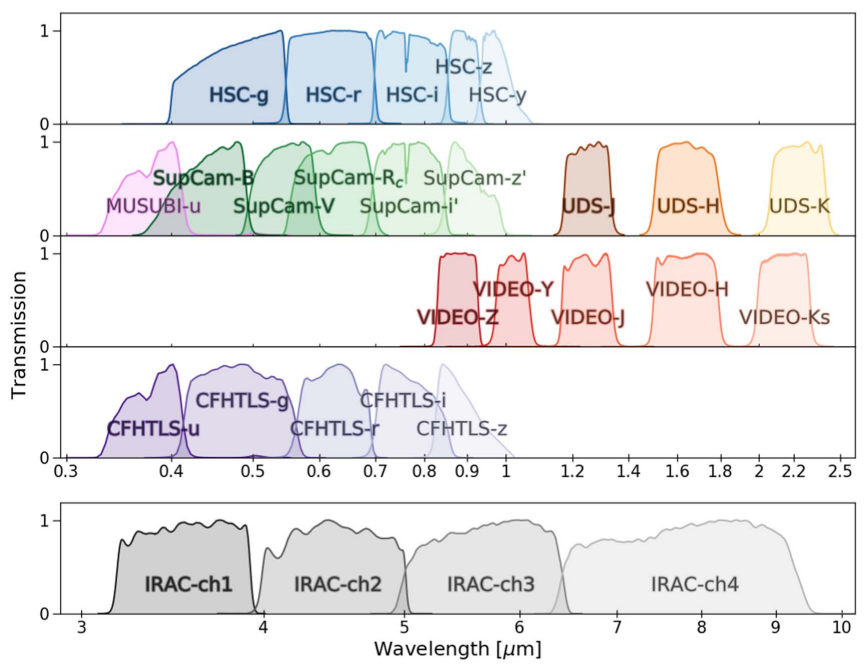

Figure 2. Transmission curves for the photometric bands included in the catalog. For clarity, all curves have been normalized to have a maximum throughput of one, thus the relative efficiencies of the individual telescopes and detectors are not shown.

\subsection{Mid-infrared Data}

The primary Spitzer-IRAC coverage at $3.6 \mu \mathrm{m}$ and $4.5 \mu \mathrm{m}$ (channel 1/2) in this field comes from the Spitzer Large Area with Hyper-Suprime-Cam (SPLASH) program (PID: 10042, PI: Capak, P. Capak et al. 2018, in preparation) which reached a depth of $\sim 6 \mathrm{hr}$ per pixel over the Hyper-Suprime-Cam field of view. Additional data from programs 90038, 80218 (S-CANDELS; Ashby et al. 2015), 80159, 80156, 70039, 61041 (SEDS; Ashby et al. 2013), and 60024 (SERVS; Mauduit et al. 2012) were also included. These only covered parts of the field, but notably SEDS reaches $\sim 12.5 \mathrm{hr}$ per pixel, while S-CANDELS reaches $\sim 51 \mathrm{hr}$ per pixel over small fractions of the field. The $5.4 \mu \mathrm{m}$ and $8.0 \mu \mathrm{m}$ (channel 3/4) data were obtained during the cryogenic mission primarily by program 40021 (SpUDS; Caputi et al. 2011), along with programs 3248, and 181 (SWIRE; Lonsdale et al. 2003). These cryogenic mission data also obtain some additional IRAC channel $1 / 2$ data.

The full details of the data processing are presented in a companion paper (P. Capak et al. 2018, in preparation). In brief, data reduction started with the corrected Basic Calibrated Data (cBCD) frames. These cBCDs have the basic calibration steps (dark/bias subtraction, flat-fielding, astrometric registration, photometric calibration, etc.) applied and include a correction for most known artifacts including saturation, column pulldown, reflections from bright off-field stars, muxstriping, and muxbleed in the cryogenic mission data. An addition correction was applied for the residual "first frame effect" bias pattern and the column pulldown effect and bright stars were also subtracted. In the warm mission the image uncertainty does not account for the bias pedestal level and so the uncertainty images need to be adjusted for this effect. Finally, the background was subtracted from the images to match it at zero across the mosaic.

The background-subtracted frames were then combined with the MOPEX ${ }^{23}$ mosaic pipeline. The outlier and box-outlier modules were used to reject cosmic rays, transient, and moving objects. The data were then drizzled onto a pixel scale

\footnotetext{
$\overline{23}$ http://irsa.ipac.caltech.edu/data/SPITZER/docs/dataanalysistools/tools/ mopex/
} 

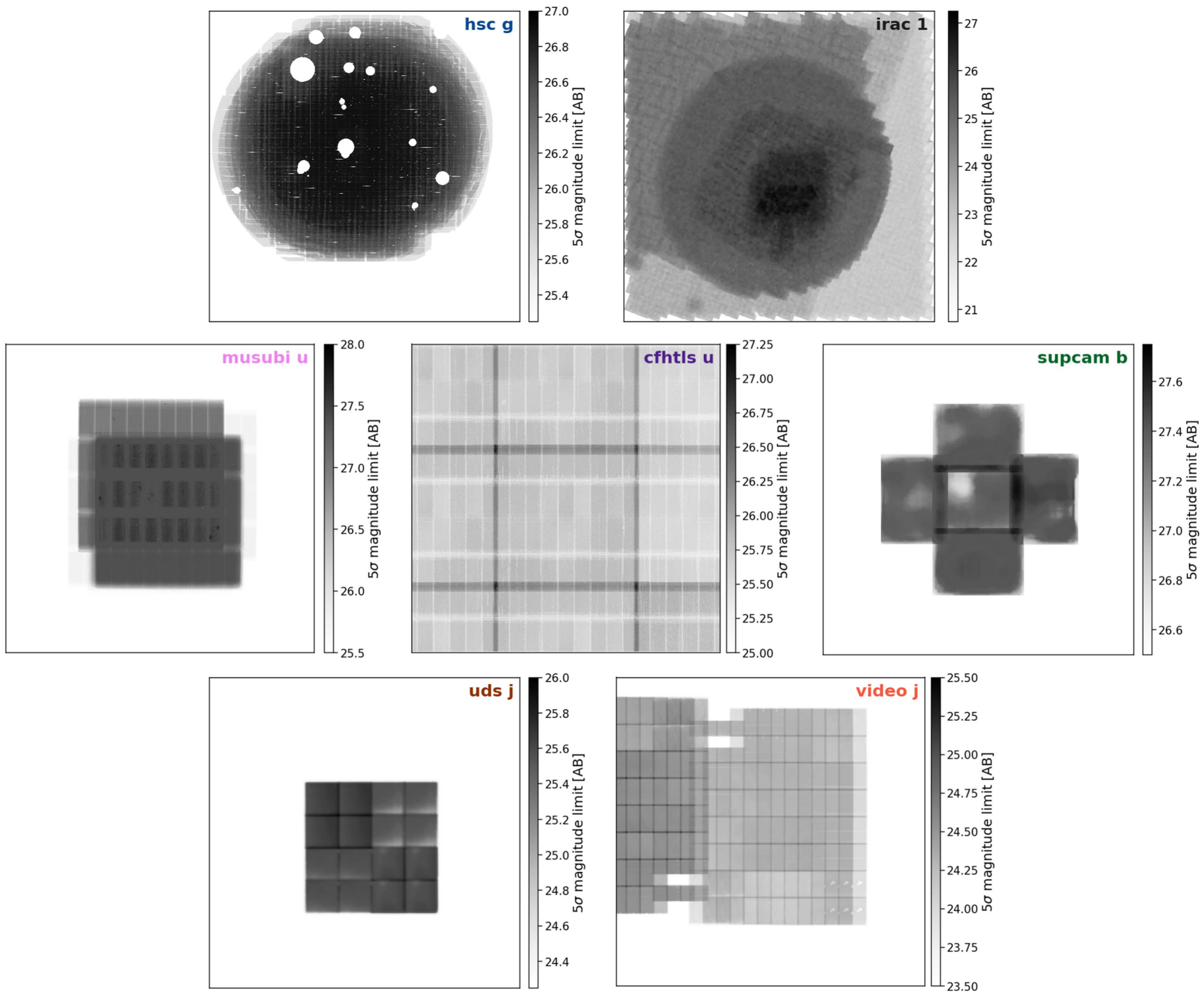

Figure 3. Maps showing the $5 \sigma$ limiting magnitude for a $2^{\prime \prime}$ circular aperture for selected bands from different surveys included in the SXDF multi-wavelength catalog. The HSC data are particularly affected by photometric artifacts around bright sources, hence a star mask specific to the HSC data is applied. Each of the panels use the same grid as Figure 1.

of 0 " $6 / \mathrm{px}$ using a "pixfrac" of 0.65 and combined with an exposure time weighting. Mean, median, coverage, uncertainty, standard deviation, and color term images were also created. The depth of the IRAC data are presented in Table 1.

\section{Data Homogenization}

\subsection{Creating Mosaics}

One of the main goals of this effort is to homogenize all data available over the SXDF area onto a single common reference frame. We create new mosaics by resampling the available imaging data from their respective sources and adjusting for the image center, pixel scale, astrometry, and photometric zero-point.

All optical and NIR data are resampled onto a single $50000 \times 50000 \mathrm{px}$ reference frame with $0{ }^{\prime \prime} 15 / \mathrm{px}$ centered at $(\alpha, \delta)=\left(02^{\mathrm{h}} 18^{\mathrm{m}} 00^{\mathrm{s}},-5^{\circ} 00^{\prime} 00^{\prime \prime}\right)$ using the $\mathrm{SWARP}^{24}$ software (Bertin et al. 2002) with the LANCZOS3 kernel. We set the

\footnotetext{
${ }^{24}$ http://www.astromatic.net/software/swarp
}

zero-point for all mosaics at $23.93 \mathrm{mag}$, equivalent to having the image in units of $\mu \mathrm{Jy}$. Corresponding weight maps are available via the data releases for the respective surveys and are also processed through SWARP, simultaneously with the science images. The HSC data processing pipeline also generates a map highlighting bright objects. We use this flag map to mask large, bright objects (stars). This star mask is taken into account when generating the HSC photometry to avoid photometric artifacts due to the bright stars in their vicinity. The mask is only applied to the HSC bands, as it is only available and valid for the HSC data.

For Suprime-Cam and CFHTLS data, we combine multiple separate pointings to get the maximum coverage and resample onto our reference frame and pixel scale. In the case of CFHTLS, even for a given band, the individual tiles have considerably different seeing and hence, we pre-process them for homogenizing the variations in the point-spread function (PSF) within the various tiles for a given band (see Section 3.3 for details). For the UDS, VIDEO, and MUSUBI- $u$ data, 


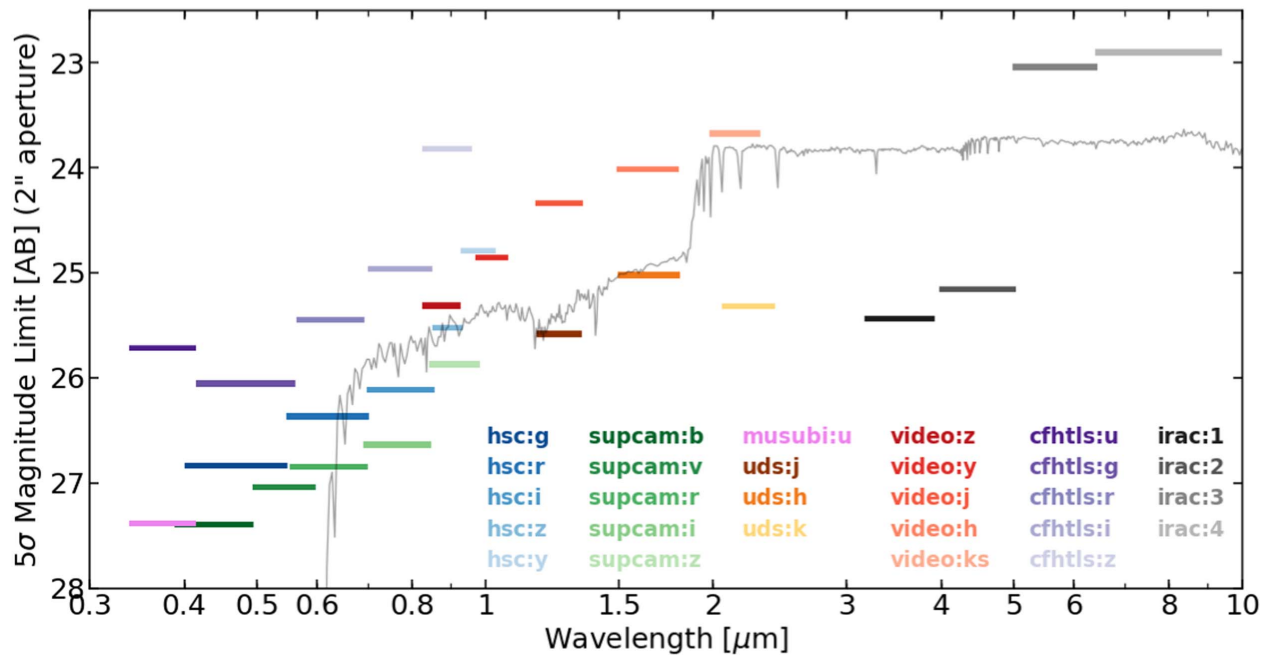

Figure 4. $5 \sigma$ limiting magnitude for a $2^{\prime \prime}$ circular aperture in each band in the SXDF multi-wavelength catalog. The limiting magnitudes are computed by measuring the sky variation in empty apertures. The galaxy template shown is a Bruzual \& Charlot (2003) single stellar population template with an age of 200 Myr and stellar mass of $10^{10} M_{\odot}$ at $z=4$.

mosaics are already available from their respective data releases and we just resample them onto our reference frame while adjusting their native pixel scales.

The IRAC data do not need resampling because the photometry for the IRAC images is measured with a different technique that performs source-fitting directly on the IRAC mosaic (with its native pixel scale of 0 !. $6 / \mathrm{px}$ ) using the optical and NIR detection as a prior (see Section 4.2 for details).

\subsection{Astrometric Corrections}

Slight astrometric deviations are expected for the observations from different instruments and surveys that are reduced and processed through different pipelines. In order to ensure a common World Coordinate System reference frame for all our imaging data, we compute an astrometric solution needed to register each image to the SDSS-DR8 catalog (Aihara et al. 2011) using SCAMP. ${ }^{25}$

The astrometric matching is done using a source catalog of reliable objects $(S / N \gtrsim 7)$ generated by running SEXTRACTOR ${ }^{26}$ (Bertin \& Arnouts 1996) on the individual mosaics. These catalogs are processed through SCAMP to match the astrometry to a user-defined reference frame. The astrometric solution is calculated by fitting a 2D polynomial (of degree 5). This allows us to remove structural offsets in the data sets as a function of $\mathrm{R}$. A. and decl. and reduce the overall scatter in both R.A. and decl. to $\lesssim 0$ ". 15 (1px) for all data sets. Figure 5 shows the reduction in the average astrometric scatter for the different data sets.

As output, SCAMP generates a FITS header with keywords (polynomial distortion parameters) containing the updated astrometric information. These parameters are compatible with SWARP. When resampling the mosaics, SWARP reads in the SCAMP solution and adjusts the astrometry according to the FITS keywords.

\subsection{PSF Homogenization}

Ground-based data are subject to variation in the pointspread function (PSF) due to the atmospheric conditions at the

\footnotetext{
25 http://www.astromatic.net/software/scamp

26 http://www.astromatic.net/software/sextractor
}

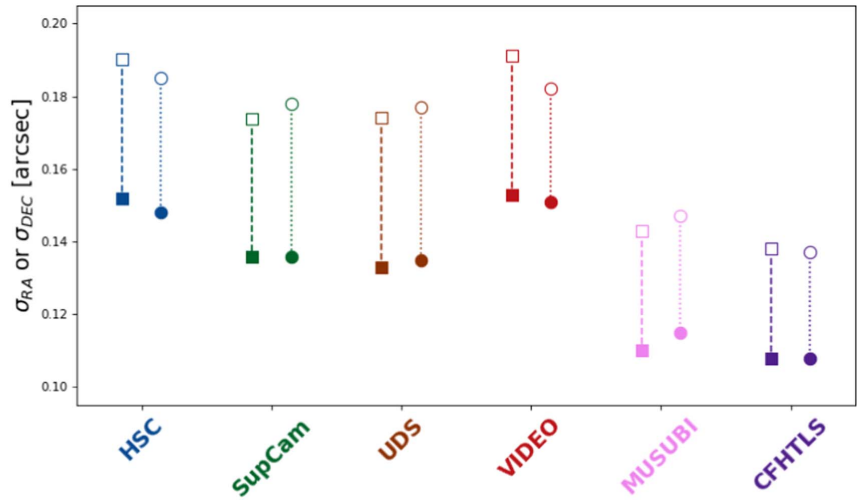

Figure 5. Average scatter in R.A. (squares) and decl. (circles) shown before (open symbols) and after (filled symbols) applying the astrometric corrections using SCAMP.

time of the observations, as well as the instrument capabilities. In order to extract accurate photometry, we homogenize the PSF across all optical and NIR bands (IRAC bands are not homogenized because their photometry is extracted using a source-fitting technique; see details in Section 4.2). The PSF homogenization process implemented here is adopted from Laigle et al. (2017).

Here, we ignore the variation in the PSF across an individual mosaic for a given band for all optical and NIR data, except CFHTLS. The PSF is relatively stable across the mosaics, and more importantly the variations across different bands are always the dominant factor compared to the variation within a single band. This has not been the case for the CFHTLS tiles, where the PSF varied considerably even for a given band. For CFHTLS observations, we specifically choose to homogenize the PSF on a tile-by-tile basis.

We use PSFEx ${ }^{27}$ (Bertin 2011) to measure the PSFs for each of our filters. First, we generate a source catalog of bright but not saturated objects by running SEXTRACTOR on the mosaics in single-image mode with a strict $>25 \sigma$ detection threshold.

$\overline{27}$ http://www.astromatic.net/software/psfex 


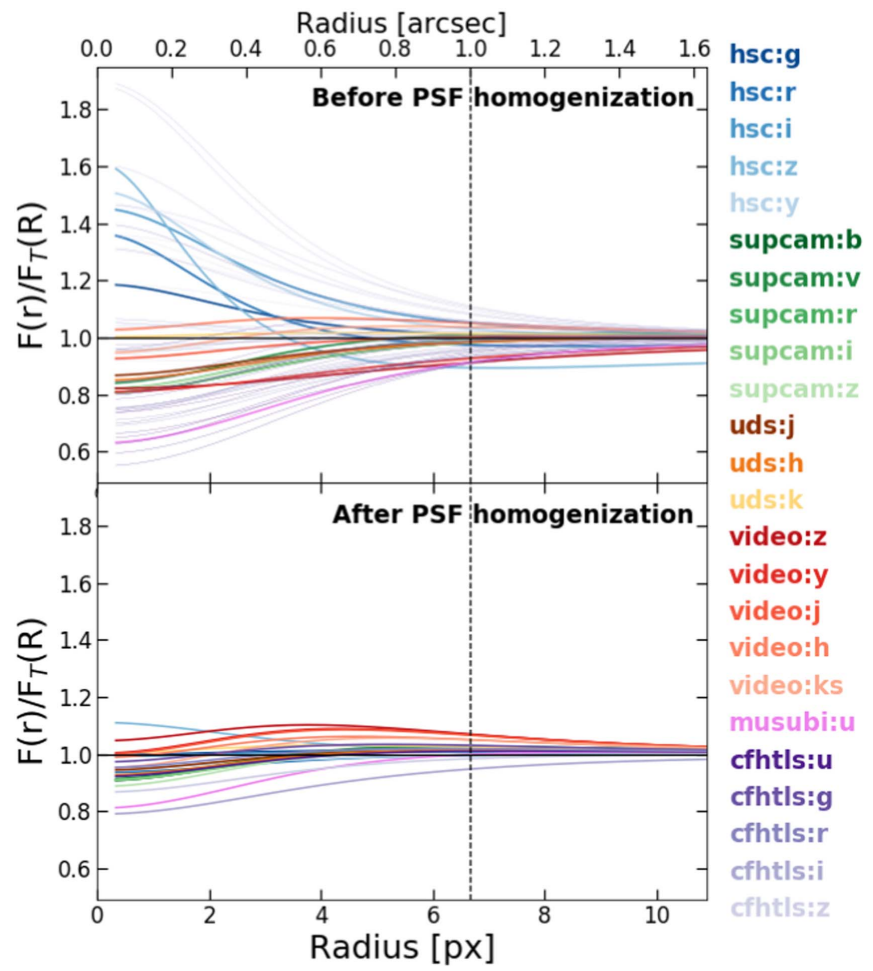

Figure 6. Curve of growth plot for the PSFs in each filter computed using PSFEx, and showing the ratio of flux within radius $(r)$ to the target PSF. The top panel shows the curves of growth before PSF homogenization; the bottom panel shows the curves after PSF homogenization. The dashed vertical line shows the $2^{\prime \prime}$ aperture.

Next, we generate a size-magnitude diagram of all detected objects. For a point source, the radius encompassing a fixed fraction of the total flux is independent of the source brightness. Consequently, point-like sources are easily identifiable on a fraction-of-light radius versus magnitude plot, as they are confined to a tight vertical locus. We only select unsaturated, point-like sources for fitting the PSF.

The PSF is modeled using Gauss-Laguerre functions, also known as the "polar shapelet" basis (Massey \& Refregier 2005). The components of the "polar shapelet" basis have explicit rotational symmetry that is useful for fitting PSFs. The global best-fit PSF for each filter is derived by $\chi^{2}$ minimization using the postage stamps of point-like sources extracted by SEXTRACTOR.

Once the best-fit PSF is obtained for each filter, we pick a "target" PSF that represents the desired PSF for all bands after homogenization. We choose a target PSF that is an average of all PSFs so as to minimize the applied convolution (see Figure 6). The target PSF is represented using a Moffat profile (Moffat 1969), as it models the inner and outer regions of the profile better than a simple Gaussian. The Moffat profile $(\mathcal{M})$ is described with two parameters $[\theta, \beta]$ as

$$
I_{r}=I_{0}\left[1+(r / \alpha)^{2}\right]^{-\beta},
$$

where $\alpha=\theta / 2 \sqrt{2^{1 / \beta}-1}, I_{0}=(\beta-1)\left(\pi \alpha^{2}\right)^{-1}$ and $\theta$ is the FWHM. We choose the Moffat profile $\mathcal{M}(\theta, \beta)=\mathcal{M}(0$ !" $7,2.8)$ as our target PSF, which is close to the average for all bands. The convolution kernels for homogenizing the PSFs are generated by PSFEx, given the parameters for our target PSF. Using the kernels, we convolve the original images to create the
Table 2

PSF Parameters from PSFEx for Optical and NIR Bands

\begin{tabular}{|c|c|c|c|c|c|}
\hline \multirow{2}{*}{$\begin{array}{l}\text { Instruments/ } \\
\text { Surveys }\end{array}$} & \multirow{2}{*}{ Filters } & \multicolumn{2}{|c|}{$\begin{array}{c}\text { Before PSF } \\
\text { Matching }\end{array}$} & \multicolumn{2}{|c|}{$\begin{array}{l}\text { After PSF } \\
\text { Matching }\end{array}$} \\
\hline & & $\overline{\theta\left({ }^{\prime \prime}\right)}$ & $\beta$ & $\overline{\theta\left({ }^{\prime \prime}\right)}$ & $\bar{\beta}$ \\
\hline \multirow[t]{5}{*}{$\overline{\mathrm{HSC}}$} & $g$ & 0.63 & 2.57 & 0.71 & 2.98 \\
\hline & $r$ & 0.55 & 2.07 & 0.73 & 2.92 \\
\hline & $i$ & 0.58 & 2.78 & 0.71 & 2.94 \\
\hline & $z$ & 0.45 & 1.64 & 0.66 & 2.72 \\
\hline & $y$ & 0.55 & 2.43 & 0.72 & 2.85 \\
\hline \multirow[t]{5}{*}{ SupCam } & $B$ & 0.80 & 3.19 & 0.77 & 3.66 \\
\hline & V & 0.80 & 3.65 & 0.77 & 3.76 \\
\hline & $R_{c}$ & 0.80 & 3.36 & 0.77 & 3.66 \\
\hline & $i^{\prime}$ & 0.80 & 3.18 & 0.76 & 3.38 \\
\hline & $z^{\prime}$ & 0.80 & 3.34 & 0.78 & 3.71 \\
\hline \multirow[t]{3}{*}{ UDS } & $J$ & 0.76 & 2.95 & 0.74 & 3.22 \\
\hline & $H$ & 0.77 & 3.00 & 0.75 & 3.30 \\
\hline & $K$ & 0.71 & 3.02 & 0.72 & 3.28 \\
\hline \multirow[t]{5}{*}{ VIDEO } & $Z$ & 0.73 & 2.25 & 0.74 & 4.84 \\
\hline & $Y$ & 0.75 & 2.40 & 0.76 & 5.14 \\
\hline & $J$ & 0.74 & 3.05 & 0.76 & 5.02 \\
\hline & $H$ & 0.73 & 3.91 & 0.75 & 4.18 \\
\hline & $K_{s}$ & 0.76 & 3.89 & 0.77 & 4.45 \\
\hline MUSUBI & $u$ & 0.89 & 2.92 & 0.81 & 3.53 \\
\hline \multirow[t]{5}{*}{ CFHTLS } & $u$ & $\ldots$ & $\ldots$ & 0.75 & 3.25 \\
\hline & $g$ & $\ldots$ & $\ldots$ & 0.74 & 3.54 \\
\hline & $r$ & $\ldots$ & $\ldots$ & 0.74 & 3.31 \\
\hline & $i$ & $\ldots$ & $\ldots$ & 0.78 & 2.68 \\
\hline & $z$ & $\ldots$ & $\ldots$ & 0.76 & 2.95 \\
\hline
\end{tabular}

PSF-homogenized set of mosaics. These are used for measuring photometry in Section 4.1.

The choice of a target PSF that is an average of all bands, as opposed to choosing the largest PSF, does result in a deconvolution for some of the bands. However, we emphasize that the convolved images are only used for flux measurement in the apertures defined by the detection image, which is made using the original unconvolved images. As such, a deconvolution is not expected to create any biases. Regardless, the differences in the PSFs are small (see Table 2) and the (de) convolution is not drastic.

Figure 6 shows the curve of growth, i.e., the flux contained within a given aperture for each PSF normalized by that of the target PSF, both before and after the homogenization. After homogenization the variation across bands is reduced to $\lesssim 5 \%$ for a $2^{\prime \prime}$ aperture. Table 2 provides the Moffat profile parameters as derived by PSFEx in each band.

\section{Multi-wavelength Catalog}

\subsection{Source Extraction}

The object detection and flux measurement are done using SEXTRACTOR. We run SEXTRACTOR in dual image mode with the detection image defined as a combination of HSC-grzy, UDS-JHK, VIDEO-YJHKs, MUSUBI- $u$, and CFHTLS-ugri. We use a $\chi^{2}$ combination in SWARP to create the detection image, which combines the input images by taking the square root of the reduced $\chi^{2}$ of all pixel values with non-zero weights. The $\chi^{2}$ combination is optimal for panchromatic detection on images taken at different wavelengths (Szalay 


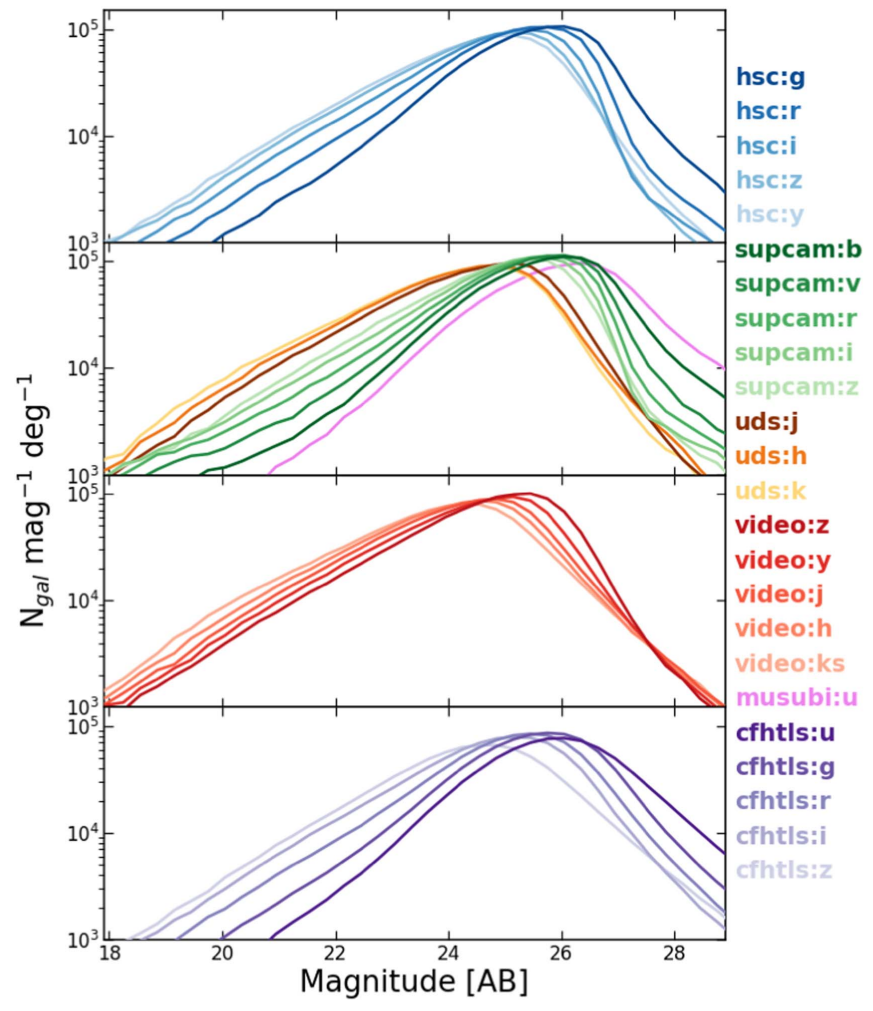

Figure 7. Number counts of sources detected using our multi-band detection image shown for each filter as a function of magnitude.

et al. 1999). The non-homogenized versions of the mosaics are used for making the detection image in order to preserve the original noise properties of the data. The $u$-band data are included to ensure the detection of even the bluest objects. We exclude the HSC- $i$ band due to excessive satellite trails that are left over from the pipeline reduction. Mosaics for the same band from different surveys are included in the detection image because they have different depths and coverage areas.

We detect $\sim 1.17$ million objects over the full mosaic area (4.2 $\mathrm{deg}^{2}$ ), with $\sim 800,000$ objects detected in the $2.4 \mathrm{deg}^{2}$ HSC-UD area. Figure 7 shows the number counts of detected sources in each filter.

The fluxes are measured on the PSF-homogenized mosaics. We optimize the SEXTRACTOR parameters to ensure that real faint sources are detected, particularly near bright sources. The parameters used for our SEXTRACTOR run are listed in Table 5. In addition to the Kron (1980) aperture (AUTO) and isophotal (ISO) magnitudes, we extract fixed-aperture fluxes for $1^{\prime \prime}, 2^{\prime \prime}$, $3^{\prime \prime}, 4^{\prime \prime}$, and $5^{\prime \prime}$ circular apertures.

We correct the fluxes for Galactic extinction using the Schlegel et al. (1998) dust maps. ${ }^{28}$ The reddening $E(B-\mathrm{V})$ due to galactic dust is queried for each object position and converted into an extinction $\left(A_{\lambda}\right)$ in each band using the Cardelli et al. (1989) extinction law. Figure 8 shows the extinction applied across the SXDF. Since the SXDF is large, the Galactic extinction varies by $A_{V} \sim 0.034$ over the field. The magnitudes and fluxes in the final catalog are corrected using these values and the Galactic reddening $E(B-V)$ values are also reported in the final catalog for each source.

\footnotetext{
28 Specifically, we use the Python implementation available at http://github. com/adrn/SFD to query the SFD dust maps.
}
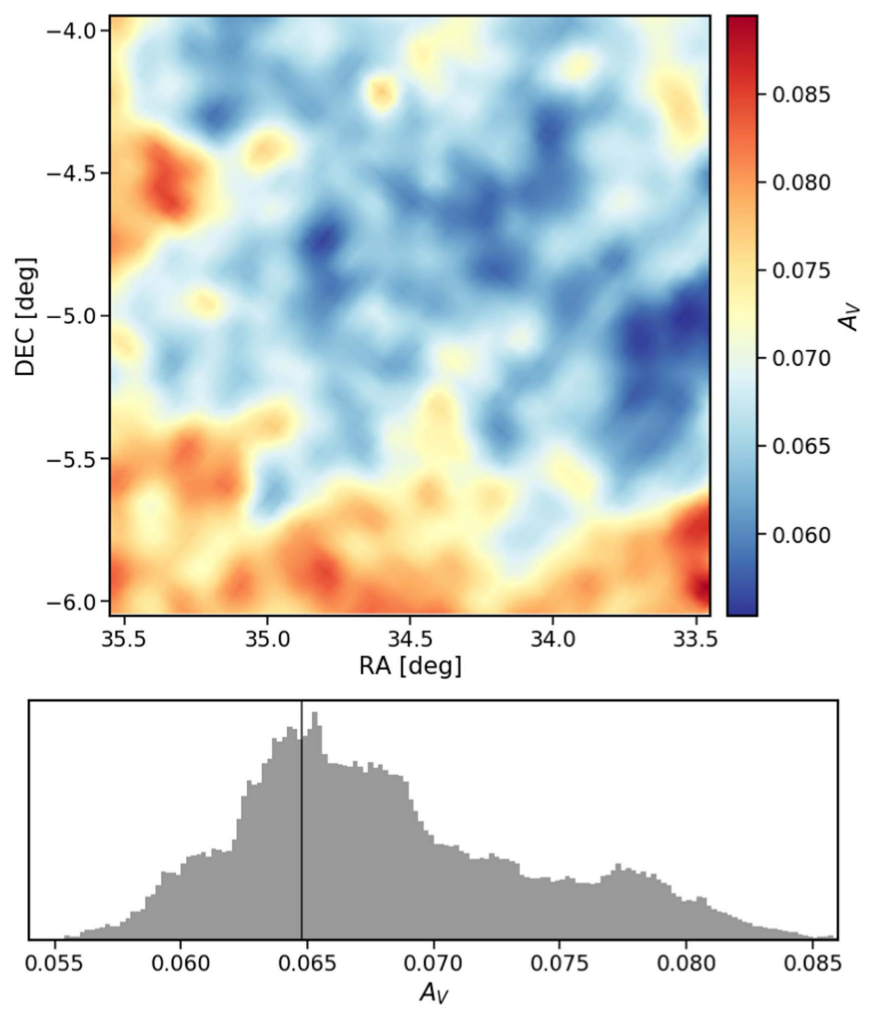

Figure 8. Galactic extinction corrected for in the SPLASH-SXDS catalog. The top panel shows the variation in the $V$-band extinction across the field. The bottom panel shows the distribution of $A_{V}$ for all the sources in the catalog.

\subsection{IRAC Photometry}

The IRAC observations have a considerably larger PSF than the optical and NIR data. In order to properly extract photometry from the high-confusion, crowded, and lowresolution IRAC data, proper deblending is necessary. We use the IRACLEAN code (Hsieh et al. 2012) as modified by Laigle et al. (2016) to measure the fluxes for the IRAC channels. IRACLEAN deblends objects and measures accurate fluxes in a low-resolution image using, as a prior, the positional and morphological properties of sources detected in a higherresolution image in a different wavelength bandpass. We use our detection image made using optical and NIR bands as the prior in IRACLEAN. For a detailed description of the various steps involved in IRACLEAN, we refer the reader to Hsieh et al. (2012).

IRACLEAN operates without the restriction that the intrinsic morphology of the source be the same in the two bandpasses. This is critical for minimizing the effect of morphological $k$-corrections when the prior and measurement bandpasses are different. However, as noted in Hsieh et al. (2012), the flux measurement can be biased when two objects are separated by less than $\sim 1$ FWHM - in such cases, the flux of the brighter object can be overestimated. The Laigle et al. (2016) modification weights each object by the surface brightness of the object in the prior image when measuring fluxes to avoid the potential bias. We refer the reader to Laigle et al. (2016), where this is described in detail.

The PSF modeling is critical to deblend sources in IRACLEAN to measure accurate photometry. However, the PSF of IRAC is asymmetric, therefore the effective PSF shape depends on the rotation and depth of individual frames that are combined to a mosaic, hence it is expected to vary as a function 
of position. We therefore derive the effective PSF for each position on the final SDXF IRAC mosaic by combining all the changes to IRAC's intrinsic point response function (PRF) caused by the mosaicking process. For this, we start with the intrinsic PRF (depending on the position of the detector) at a native scale of 1 "! 22 and oversample by a factor of 100 . The PRFs are rotated by the position angle of their corresponding individual frames and then run through the mosaicking process to obtain the final, combined PSF at each pre-defined grid point on the final mosaic.

Instead of using a static PSF across the full mosaic, IRACLEAN is modified to read in all the PSFs derived on a $30^{\prime \prime}$ grid over the mosaic. When performing the deblending algorithm for individual sources, the routine looks up the nearest grid point to the object position and uses the PSF associated with it. This functionality allows IRACLEAN to fully account for the variations in the PSF as a function of position on the large-area mosaic.

The segmentation map created from the optical and NIR detection image (described in Section 4.1) is used as the prior for measuring IRAC photometry in IRACLEAN. The segmentation map is identical in size and dimension to the detection image, with pixels attributed to each detected object set to the object's identification number. Since the segmentation map and the IRAC mosaics need to be on the same pixel scale for running IRACLEAN, we rebin the segmentation map to the resolution of the IRAC images $\left(00^{\prime \prime} 6 / \mathrm{px}\right)$. The rebinned segmentation map is created by taking the mode of all the sub-pixels entering the rebinned pixel. We take additional measures to preserve objects that would occupy less than a pixel in the rebinned segmentation map but do not have any other nearby overlapping sources. Specifically, if a rebinned pixel assigned as background (from the calculation of the mode) had a detected object in the original segmentation map, the rebinned pixel is instead assigned to the detected object. This is critical for preserving isolated objects that are compact in the optical/NIR detection image but could still be extended in the IRAC channels.

With this prescription, the rebinned segmentation map could still potentially miss sources that are compact (less than 1 IRAC pixel) and adjacent to another bright object. Hence, these objects are not guaranteed to be recovered even though the IRAC mosaic has coverage at their positions. Only $0.2 \%$ (1474) of the objects from our optical and NIR detection image fall into this category. In order to distinguish between IRAC sources lost due to a lack of IRAC coverage and those due to rebinning of the segmentation map, we specify a source extraction flag (SE_FLAGS_irac_* ) for the IRAC photometry in the catalog. Objects lost due to the rebinning of the segmentation map are assigned a flag of 1 , whereas those not recovered due to a lack of coverage are given a flag of 2 .

Lastly, in order to save computation time, we parallelize the photometry measuring process by running IRACLEAN on cutouts of the full mosaic. The IRAC mosaic is split into $1000 \times 1000 \mathrm{px}\left(600^{\prime} \times 600^{\prime}\right)$ tiles with an overlap of $977^{\prime \prime} 5$ to avoid edge effects. IRACLEAN is run for each tile using the surface brightness weighting parameter of $n=0.3$ and an aperture size of 1 ." $8 \times 1$ " 8 to measure the flux ratios between the sources and PSFs for the deblending procedure. The final photometric catalog includes the total fluxes and associated errors for each object present in the segmentation map as measured by IRACLEAN. For objects with fluxes below $1 \sigma$, their magnitudes are set to the corresponding $1 \sigma$ upper limits.

\subsection{Photometric Errors and Magnitude Upper Limits}

Since the optical and NIR images undergo multiple processing steps, it is critical to ensure that the photometric errors are propagated correctly.

\subsubsection{SWARP Photometric Errors}

All optical and NIR images used for measuring photometry are resampled onto a common reference frame. This step involves adjusting the pixel scale of the original images to a common 0 !" $15 /$ px. While SWARP is expected to scale the science images and the weight maps in a consistent fashion, we test the photometric error properties explicitly before and after processing through SWARP.

For this test, source catalogs are generated in single-image mode from the original and SWARP-processed images using SEXTRACTOR with the same parameters. We first compare the measured fixed-aperture fluxes and find them to agree. We compare the errors on aperture photometry for bright sources and find the errors to be systematically offset as a function of the original pixel scale. The photometric errors are underestimated for cases in which the original pixel scale is finer than the final pixel scale, whereas they are overestimated for cases in which the original pixel scale is coarser.

Figure 9 shows the correction factor in each band needed for the photometric errors to be consistent before and after the SWARP resampling process. The correction factor scales in almost the same way as the ratio of pixel scales (original: resampled). As the first step, we adjust the photometric errors in each band to correct for this systematic.

\subsubsection{Sky Noise Properties and Survey Depth}

A more appropriate description of the photometric errors is derived from the sky noise. While the weight maps account for errors arising from instrumental effects and observation strategies, measuring the sky variation has the added benefit of accounting for undetected faint objects. The errors computed by SEXTRACTOR account for the provided weight maps. However, since we perform photometry on the PSFhomogenized images, the errors measured from SEXTRACTOR are not precise due to the additional unaccounted correlated noise. When convolving the mosaics with the homogenization kernel to match the PSFs, the sky noise properties are altered.

In order to accurately measure the sky noise, we compute the flux variation in random empty sky apertures for each band from the unconvolved mosaics. The sky noise properties are dependent on two major factors: the aperture size and the image depth. We measure the sky noise in $1^{\prime \prime}, 2^{\prime \prime}, 3^{\prime \prime}, 4^{\prime \prime}$, and $5^{\prime \prime}$ circular apertures to quantify the dependence on aperture size. We use the value of the weight maps as a proxy for image depth, since the mosaic's exposure time information is encoded in the weight maps. Ultimately, the sky noise is computed as a function of the aperture size and image depth. We use the SEXTRACTOR segmentation map to avoid sources when placing the random sky apertures and measure their fluxes using PHOTUTILS ${ }^{29}$ (Bradley et al. 2016). The sky variation is measured by fitting a Gaussian to the distribution of fluxes in the sky apertures in bins of the value of the weight map at the location of the source. We explicitly only fit the half of the sky flux distribution that is below its peak.

\footnotetext{
${ }^{29}$ http://photutils.readthedocs.io/
} 


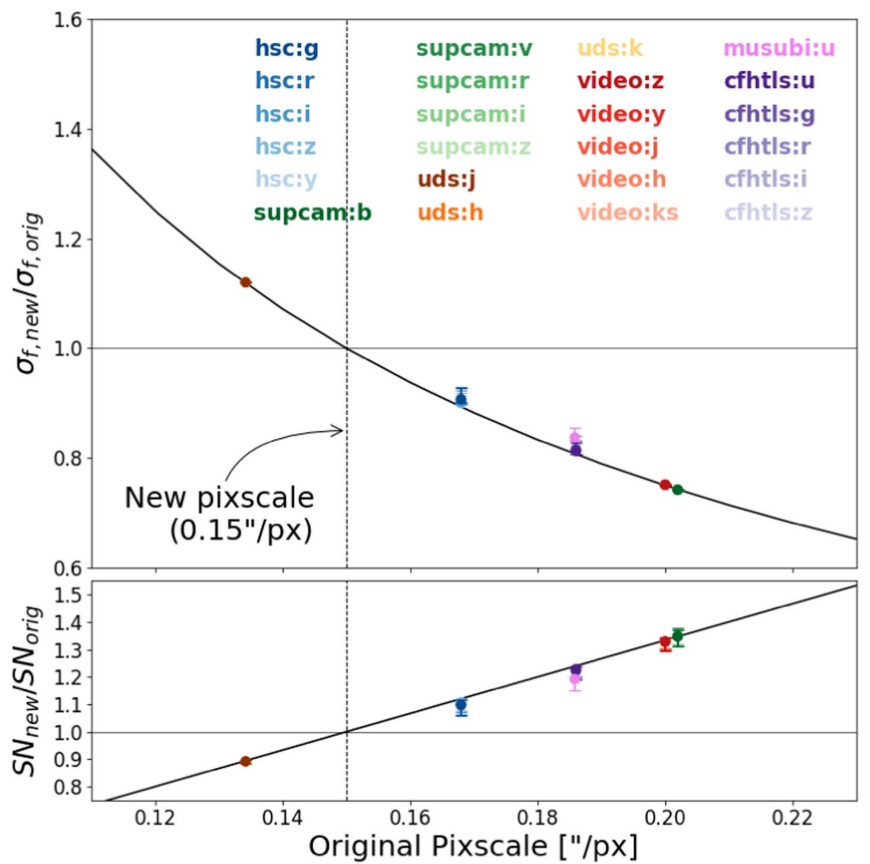

Figure 9. Comparison of the photometric errors before and after resampling images through SWARP. The key adjustment made when resampling is changing the native pixel scale to match that of our reference frame $(0$. ! $15 / \mathrm{px})$. The photometric errors are systematically offset as a function of the ratio of native pixel scale to the resampled one.

The sky variation represents the faintest flux level (magnitude) at which a source can be detected in the mosaic. Thus, we can use the computed sky noise to define a $1 \sigma$ limiting magnitude for our filters. Figure 3 shows the $5 \sigma$ limiting magnitude for a $2^{\prime \prime}$ circular aperture over the full mosaic for an example filter from each data set included in this work. A representative $5 \sigma$ depth for each filter is listed in Table 1 and shown in Figure 4. Only for the fixed-aperture magnitudes reported in the photometric catalog, if the object's flux is below the corresponding sky noise value for the given aperture and image depth at the object's position, the magnitude of that object is set to the $1 \sigma$ upper limit. The photometric redshift computation uses the measured fluxes and does not utilize the upper limits (which are only applied to the magnitudes).

\subsubsection{Correcting Photometric Errors}

We compute a correction factor for adjusting the SEXTRACTOR errors to match the sky variation. The correction factor is computed as the ratio between the sky variation measured from the random sky apertures and the median of the SEXTRACTOR errors. This factor is computed after applying the correction from Section 4.3.1. See Appendix B for a brief discussion of this procedure and see Table 6 for the applied correction factors.

Although this correction factor can be computed for each object in each of the filters, there is no physical motivation to apply the correction on a source-by-source basis. All processes affecting the photometric errors (e.g., resampling, convolution, etc.) are performed on the full mosaic and hence should not have different impacts on an individual object basis. Ideally, in order to correct for these effects, a single correction factor over the full mosaic should suffice. However, we expect that areas of the mosaics with different depths are affected differently. Considering that most of our filters have varying depths across the image due to stacking different pointings, we choose to compute a separate factor when the image depth changed significantly. The computed correction is uniform over two regions for the Suprime-Cam $B V R_{c} i^{\prime} z^{\prime}$ and VIDEO $J$ mosaics, and three regions for the MUSUBI $u$-band mosaic.

We compute the correction factors for the fixed apertures $\left(1^{\prime \prime}, 2^{\prime \prime}, 3^{\prime \prime}, 4^{\prime \prime}, 5^{\prime \prime}\right)$. For AUTO and ISO fluxes, we calculate the correction factor by interpolating between the fixed-aperture sizes. We use the Kron (1980) radius and number of pixels in the isophotal aperture to estimate the size of the AUTO and ISO apertures, respectively. The errors on fluxes and magnitudes in the catalog have this correction already applied.

\subsection{Ancillary Data Sets}

Simpson et al. (2006) covered the SXDF with the Very Large Array (VLA) to obtain radio imaging at $1.4 \mathrm{GHz}$. Their catalog lists radio sources covering $0.8 \mathrm{deg}^{2}$ on the SXDF down to a peak flux density of $100 \mu \mathrm{Jy}$ beam $^{-1}$. Their synthesized beam has a roughly elliptical shape characterized by $\sim 5^{\prime \prime} \times 4$ !. They identify optical counterparts to the radio sources using the $B V R_{c} i^{\prime} z^{\prime}$ Suprime-Cam images from Furusawa et al. (2008). For detailed description of the process of identifying the optical counterparts, we refer the reader to Simpson et al. (2006). We use their identified optical counterparts and match them to our photometric catalog. The $1.4 \mathrm{GHz}$ fluxes from the radio catalog are included in our final photometric catalog.

Akiyama et al. (2015) presented a catalog of X-ray sources over $1.3 \mathrm{deg}^{2}$ centered on the SXDF using XMM-Newton. The full area is covered with one central $30^{\prime}$ diameter, $100 \mathrm{ks}$ exposure along with six flanking fields with 50ks exposures. The details of the observations and data processing are described in Ueda et al. (2008). Akiyama et al. (2015) selected counterparts to the X-ray sources in the Suprime-Cam $R$-band, IRAC $3.6 \mu \mathrm{m}$ channel, Near$\mathrm{UV}$, and $24 \mu \mathrm{m}$ source catalog image using a likelihood ratio analysis. We refer the reader to Akiyama et al. (2015) for a detailed description of the counterpart-identifying procedure. Using the positional information of these optical counterparts, we match and add the X-ray information for their objects to our catalog.

\section{Photometric Redshifts and Physical Properties}

\subsection{Photometric Redshifts}

We compute the photometric redshifts for all objects in the catalog using LEPHARE ${ }^{30}$ (Arnouts et al. 1999; Ilbert et al. 2006). Object fluxes are used for the calculation of photometric redshift rather than object magnitudes, allowing for a robust treatment of faint objects and objects undetected in one or more filters. For faint sources, even negative fluxes are physically meaningful when included with the appropriate errors; while working with magnitudes upper limits are needed, which require a modification in the $\chi^{2}$ minimization algorithm (Sawicki 2012).

Photometric redshift estimates are more accurate when galaxy colors are computed using fixed-aperture photometry, rather than pseudo-total magnitudes like AUTO magnitudes. The latter ones assume a Kron (1980) aperture, and may be much noisier, especially for the faintest objects, due to the variable nature of this profile (see Hildebrandt et al. 2012; Moutard et al. 2016). On the other hand, fixed-aperture magnitudes are more appropriate for measuring colors of galaxies; however, these are affected by the variations in the PSF across different bands. In our case, the issue of

\footnotetext{
${ }^{30}$ http://www.cfht.hawaii.edu/ arnouts/lephare.html
} 
Table 3

Systematic Offsets between the Measured 2" Fluxes and Library Templates as Computed by LEPHARE

\begin{tabular}{|c|c|c|}
\hline $\begin{array}{l}\text { Instrument/ } \\
\text { Survey }\end{array}$ & Filters & $\begin{array}{c}\text { Systematic } \\
\text { Offset }\left(2^{\prime \prime}\right)[\mathrm{mag}]\end{array}$ \\
\hline$\overline{\mathrm{HSC}}$ & $\begin{array}{l}g \\
r \\
i \\
z \\
y\end{array}$ & $\begin{array}{l}-0.065 \\
-0.041 \\
-0.023 \\
-0.063 \\
-0.046\end{array}$ \\
\hline SupCam & $\begin{array}{c}B \\
V \\
R_{c} \\
i^{\prime} \\
z^{\prime}\end{array}$ & $\begin{array}{r}-0.047 \\
-0.025 \\
-0.124 \\
0.041 \\
0.061\end{array}$ \\
\hline UDS & $\begin{array}{l}J \\
H \\
K\end{array}$ & $\begin{array}{r}0.055 \\
0.023 \\
-0.003\end{array}$ \\
\hline VIDEO & $\begin{array}{l}Z \\
Y \\
J \\
H \\
K_{s}\end{array}$ & $\begin{array}{r}-0.046 \\
-0.035 \\
0.011 \\
0.030 \\
-0.061\end{array}$ \\
\hline MUSUBI & $u$ & 0.043 \\
\hline CFHTLS & $\begin{array}{l}u \\
g \\
r \\
i \\
z\end{array}$ & $\begin{array}{l}0.202 \\
0.098 \\
0.128 \\
0.045 \\
0.138\end{array}$ \\
\hline IRAC & $\begin{array}{l}\text { ch. } 1 \\
\text { ch. } 2 \\
\text { ch. } 3 \\
\text { ch. } 4\end{array}$ & $\begin{array}{l}0.100 \\
0.100 \\
\ldots \\
\ldots\end{array}$ \\
\hline
\end{tabular}

band-to-band PSF variations is solved by the PSF homogenization (Section 3.3). We use the 2 " aperture for measuring photometric redshifts because from our testing with available spectroscopic redshifts, we find that it performs better than the $1^{\prime \prime}, 3^{\prime \prime}, 4^{\prime \prime}$, and $5^{\prime \prime}$ apertures at recovering the redshifts.

Aperture photometry is available for the optical and NIR bands from SEXTRACTOR; however, for IRAC channels we only perform measurement of the total flux. IRAC total fluxes need to be scaled to match the aperture fluxes before performing SED fitting to measure the photometric redshifts. In order to adjust the total IRAC fluxes, we compute an offset factor that converts between the aperture and total magnitudes. Since the photometry is performed on PSF-homogenized images, the offset between the aperture and total magnitudes is expected to be the same across all bands. We check that the computed photometric offsets do not depend on other galaxy properties. We find no correlation with respect to the galaxy colors or magnitudes. Using multiple bands for calculating the offset also makes it more robust than using a single band. Following the treatment from Moutard et al. (2016), we compute a single multiplicative offset between the AUTO and aperture fluxes for each object as

$$
o=\frac{1}{\sum_{\text {filter } i} w_{i}} \times \sum_{\text {filter } i}\left(\frac{f_{\mathrm{AUTO}, i}}{f_{\mathrm{APER}, i}} \cdot w_{i}\right),
$$

where the weights $w_{i}$ are defined as

$$
w_{i}=\frac{1}{\sigma_{f_{\mathrm{AUTO}, i}}^{2}+\sigma_{f_{\mathrm{APER}, i}}^{2}} .
$$

The offsets for each object are provided in the final catalog.

The template library used in LePhare for photometric redshift calculation is similar to that used for the COSMOS field (Ilbert et al. 2009, 2013; Laigle et al. 2016). The template set consists of 31 templates, which includes 19 templates of spiral and elliptical galaxies from Polletta et al. (2007) and 12 templates of young blue-star-forming galaxies from the Bruzual \& Charlot (2003) model (BC03). We also include two additional extinction-free $\mathrm{BC} 03$ templates with an exponentially declining star-formation history (SFH) with a short timescale of $\tau=0.3 \mathrm{Gyr}$ and metallicities $(Z=0.008$ and $Z=0.02=Z_{\odot}$ ). As detailed in Ilbert et al. (2013), these improve the photometric redshifts for passive galaxies at $z>1.5$ (Onodera et al. 2012), which are not well represented in the Polletta et al. (2007) library. The two additional BC03 templates are sampled for 22 ages between $0.5 \mathrm{Gyr}$ and $4 \mathrm{Gyr}$.

Dust extinction is left as a free parameter and allowed to vary within $0 \leqslant E(B-V) \leqslant 0.5$. A variety of dust extinction laws is considered: Prevot et al. (1984), Calzetti et al. (2000), and a modified version of the Calzetti et al. (2000) law that includes contributions from the $2175 \AA$ bump (Fitzpatrick 1986) as proposed by Massarotti et al. (2001). No extinction is added for templates of earlier types than S0. Also, since the Sa and $\mathrm{Sb}$ templates from Polletta et al. (2007) are empirical and already include dust, no extinction is allowed for these templates either.

In addition to the galaxy templates, we also include stellar templates from Bixler et al. (1991), Pickles (1998), Chabrier et al. (2000), and Baraffe et al. (2015). In particular, we include a large number of low-mass stars of spectral classes $\mathrm{M}$ through $\mathrm{T}$ from Baraffe et al. (2015). These stellar templates extend out to $\lambda>2.5 \mu \mathrm{m}$ and thus help distinguish between distant galaxies and brown dwarf stars (Wilkins et al. 2014; Davidzon et al. 2017).

Expected fluxes in each band for all the templates are computed on a grid of $0<z<6$. Contribution from emission lines is accounted for in the flux computation using an empirical relation between the UV luminosity and the emission lines fluxes as described in Ilbert et al. (2009). The photometric redshifts (Z_BEST) are derived by $\chi^{2}$ minimization.

One of the critical steps in computing photometric redshifts is evaluating the systematic offsets between the template and observed fluxes (Ilbert et al. 2006). These systematic offsets can be measured using known spectroscopic redshifts. For this galaxy subsample, the redshift is fixed at the spectroscopic value and then LePhare performs the template fitting in an iterative fashion, adding systematic offsets to each band until $\chi^{2}$ minimization convergence is reached. The resulting offsets (Table 3 ) are applied to the input catalog when computing the photometric redshifts.

We exclude IRAC ch. 3 and 4 when measuring the photometric redshifts due to their high systematic offsets $(0.4 \mathrm{mag})$. These systematic offsets values are likely due to the limitations of the template library, specifically the lack of dust emission redward of rest-frame $2 \mu \mathrm{m}$. A cross-check with external Spitzer catalogs confirms that the photometric calibration in ch. 3 and 4 is accurate to $2 \%$. This issue will likely be resolved with future JWST data that will provide high signal-to-noise ratio templates at these long wavelengths. 

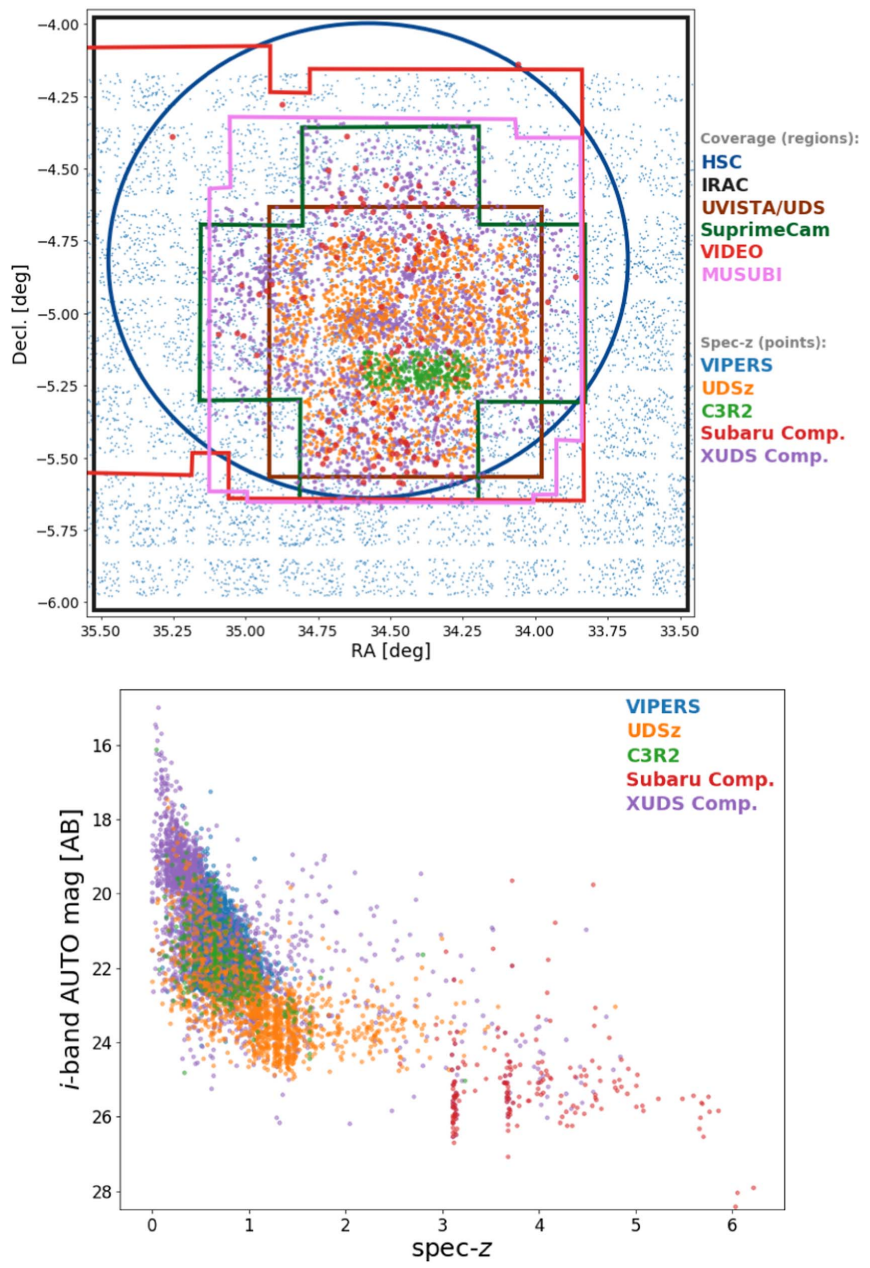

Figure 10. Positions (top panel) and the redshift-magnitude distribution (bottom panel) of the sources used to refine and verify the performance of the photometric redshifts. These galaxies have a robust spectroscopic redshift measurement available from the various surveys described in Section 5.1.1.

\subsubsection{Comparing Photometric and Spectroscopic Redshifts}

Numerous spectroscopic surveys cover the SXDF and hence a large number of objects have spectroscopic redshifts available. Table 4 lists the properties of the various spectroscopic samples that are included in the catalog.

1. The VIMOS Public Extragalactic Redshift Survey (VIPERS; Garilli et al. 2014; Guzzo et al. 2014; Scodeggio et al. 2016) covers the SXDF, and measures galaxy spectra using VIMOS on the VLT. We match the VIPERS catalog to our photometric catalog and only retain objects with highly secure spectroscopic redshifts with confidence $>99 \%$ (quality flag $>=3$ ). We exclude objects identified as AGNs in the VIPERS catalog. The VIPERS catalog contributes spectroscopic redshifts for 8451 objects.

2. The UKIDSS Ultra-Deep Survey (UDSz; Bradshaw et al. 2013; McLure et al. 2013) obtained spectra for over $3000 K$-selected galaxies using VIMOS and FORS2 instruments on the VLT. These galaxies span $1.3<z<1.5$ over a $0.6 \mathrm{deg}^{2}$ on the UDS field (part of the SXDF) down to a limit of $K=23$. The UDSz catalog contributes spectroscopic redshifts for 1489 sources in our catalog.
Table 4

Spectroscopic Redshift Samples for Objects in the SPLASH-SXDF Catalog

\begin{tabular}{lcccc}
\hline \hline $\begin{array}{l}\text { Spectroscopic Survey/ } \\
\text { Reference }\end{array}$ & $\begin{array}{c}\text { Number } \\
\text { of spec-z }\end{array}$ & $z_{\text {med }}$ & $z_{\text {range }}$ & $\begin{array}{c}i_{\text {med }} \\
{[\mathrm{mag}]}\end{array}$ \\
\hline VIPERS & 8434 & 0.67 & {$[0.07,1.71]$} & 20.9 \\
UDSz & 1486 & 1.09 & {$[0.00,4.79]$} & 23.1 \\
C3R2 & 319 & 0.65 & {$[0.04,3.24]$} & 21.8 \\
Subaru compilation & 183 & 3.71 & {$[2.56,6.21]$} & 25.3 \\
X-UDS compilation & 2111 & 0.44 & {$[0.00,4.84]$} & 20.6 \\
\hline
\end{tabular}

3. The Complete Calibration of the Color-Redshift relation survey (C3R2; Masters et al. 2017) is obtaining spectroscopic redshifts for a large sample of targeted sources in COSMOS, SXDF, and EGS using Keck (DEIMOS, LRIS and MOSFIRE), the Gran Telescopio Canarias (GTC; OSIRIS), and the Very Large Telescope (VLT; FORS2 and KMOS). A sample of 320 galaxies in the SXDF from our catalog has secure spectroscopic redshifts (quality flag $>=3$ ) available from the C3R2 survey.

4. Subaru compilation: This sample contributes spectroscopic redshifts for 122 objects in the catalog. These are narrowband- and broadband-selected objects in the SXDF provided by M. Ouchi (2018, private communication) obtained from the Subaru and Magellan telescopes. This includes objects from Ouchi et al. (2005, 2008, 2010), Saito et al. (2008), Curtis-Lake et al. (2012), Matsuoka et al. (2016), Momcheva et al. (2016), Wang et al. (2016), Ono et al. (2017), Pâris et al. (2017), Shibuya et al. (2017), R. Higuchi et al. (2018, in preparation), and Y. Harikane et al. (2018, in preparation).

5. X-UDS compilation: This sample includes spectroscopic redshifts for 2094 catalog sources. The X-UDS compilation consolidated spectroscopic redshifts from Yamada et al. (2005), Simpson et al. (2006), Geach et al. (2007), van Breukelen et al. (2007), Finoguenov et al. (2010), Akiyama et al. (2015), and Santini et al. (2015), as well as the NASA/IPAC Extragalactic Database (NED). The list provides the highest-resolution and/or best-quality spectroscopic redshift available from the references for a source.

We assemble a sample of 12,342 galaxies with reliable spectroscopic redshifts covering the full range of $0<z<6$. Figure 10 shows the distribution in the sky as well as the redshiftmagnitude distribution of the full sample of galaxies with spectroscopic redshifts. Of these, we select 8,647 galaxies that are within the HSC-UD area and have proper wavelength coverage (particularly in the NIR) to use as the calibration sample for the photometric redshifts. We quantify the performance of the photometric redshifts using two statistical measures: the normalized median absolute deviation ${ }^{31}\left(\sigma_{\mathrm{NMAD}}\right.$; Hoaglin et al. 1983) and the outlier fraction $(\eta=|\Delta z| /(1+z)>0.15)$.

Figure 11 shows a comparison of the photometric and spectroscopic redshifts for our sample. We find excellent agreement between the two over the full redshift range, with a computed $\sigma_{\mathrm{NMAD}}$ of 0.023 and an outlier fraction $(\eta)$ of $3.2 \%$ for sources within the HSC-UD area. Outside the HSCUD area where the coverage is limited, the performance of the

\footnotetext{
$\overline{31} \sigma_{\mathrm{NMAD}}=1.48 \times$ median $\left|\frac{\Delta z-\operatorname{median}(\Delta z)}{1+z_{\mathrm{spec}}}\right|$.
} 


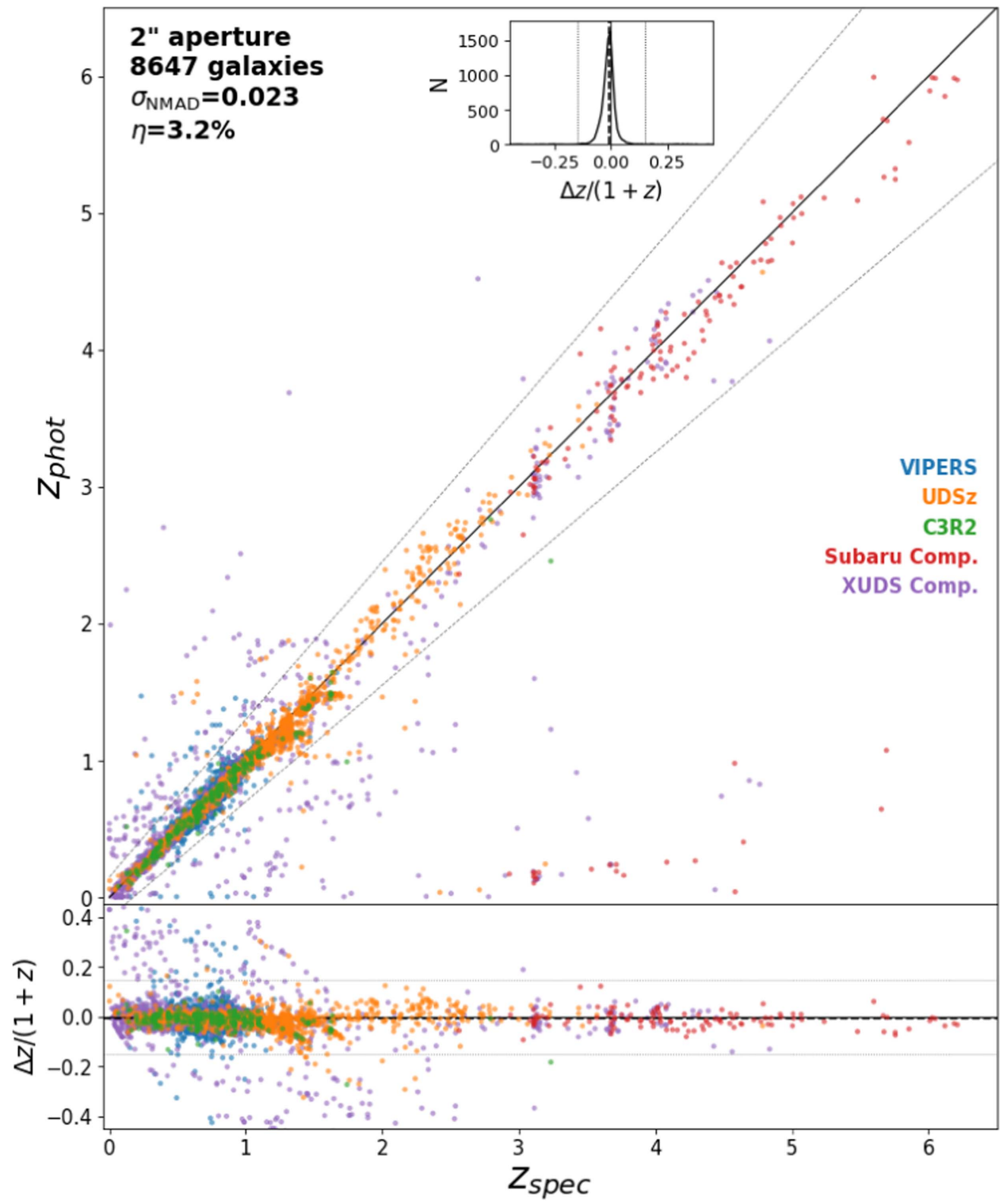

Figure 11. Comparison of the performance of the photometric redshifts in the SXDF catalog for sources inside and outside the HSC-UD area. We find a $\sigma_{\mathrm{NMAD}}=0.023$ and an outlier fraction $(\eta=|\Delta z| /(1+z)>0.15)$ of $3.2 \%$. The various colors indicate the different spectroscopic surveys that are included in the calibration sample (described in Section 5.1.1. The inset shows the distribution of the fractional differences between the photometric and spectroscopic redshifts. The dashed line in the bottom panel and in the inset shows the median value. The dotted lines show the outlier criterion: $z_{\text {phot }}=z_{\text {spec }} \pm 0.15\left(1+z_{\text {spec }}\right)$. Only objects covered within the HSC footprint are compared, since these have adequate optical and NIR coverage.

photometric redshifts drops slightly $\left(\sigma_{\mathrm{NMAD}}=0.037\right)$. as shown in Figure 12. The majority of the outliers are faint sources $\left(m_{i}>24\right)$, as evident in Figure 13, which shows the comparison as a function of the $i$-band magnitude. We find the median of the marginalized probability distribution function (Z_MED) to perform better as the photometric redshift estimator than the overall best template fit (Z_BEST). Moreover, the errors on Z_MED as reported by the $68 \%$ confidence interval (Z_MED_L68 and Z_MED_U68) are more robust. Z_MED is not estimated for sources where the marginalized probability distribution is not well behaved (these make up for $<2 \%$ of all the sources in the catalog).

\subsection{Star/Galaxy Classification}

When running LePhare for measuring the photometric redshifts, we allow for both stellar and galaxy libraries.
Comparing the best-fit solutions from each library for an object allows us to flag objects as stars. Particularly, we flag objects as stars if the $\chi_{\text {star }}^{2}<\chi_{\text {gal }}^{2}$, with a further restriction that the object is in the $B z K$ stellar sequence $(z-K s<$ $(B-z) * 0.3-0.2)$. We emphasize that since the $B$-band data from Subaru Suprime-Cam do not cover the full area of our mosaic, this classification is not available for all the sources in the catalog. Only objects that are within the Suprime-Cam footprint are classified.

Figure 14 shows the $B z K$ color-color diagram for all sources in the catalog color-coded according to their photometric redshift. As evident from the figure, the $B$-band dropouts occupying the top left part of the distribution are predominantly high-redshift $(z \gtrsim 3)$ galaxies, while galaxies with bluer $z-K$ colors are at lower redshifts. The 6,364 objects flagged as stars according to the criterion specified above are shown in black. 


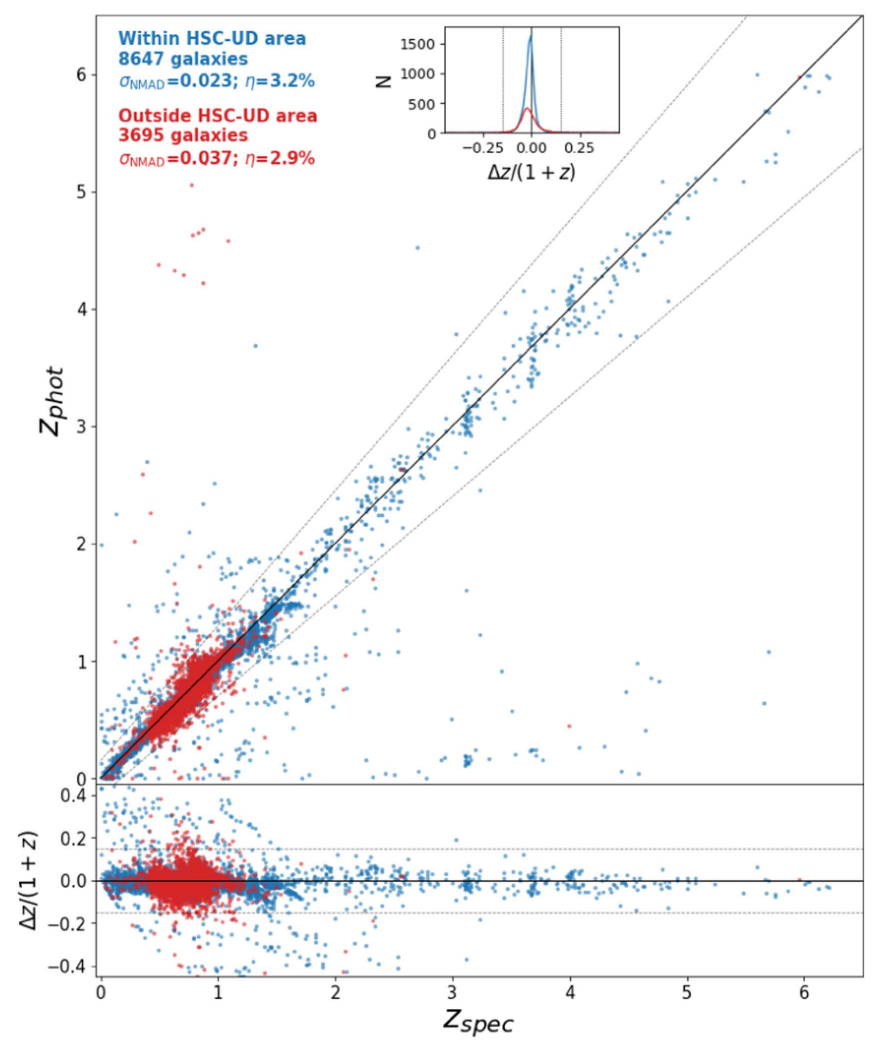

Figure 12. Performance of the photometric redshifts in the SXDF catalog compared for sources inside and outside of the HSC-UD area. Since the coverage in depth as well as wavelength is limited outside the HSC-UD area, the performance of the photometric redshifts drops slightly to $\sigma_{\mathrm{NMAD}}=0.037$. The lower outlier fraction is mainly due to the limited redshift coverage of the calibration sample outside the HSC-UD area.

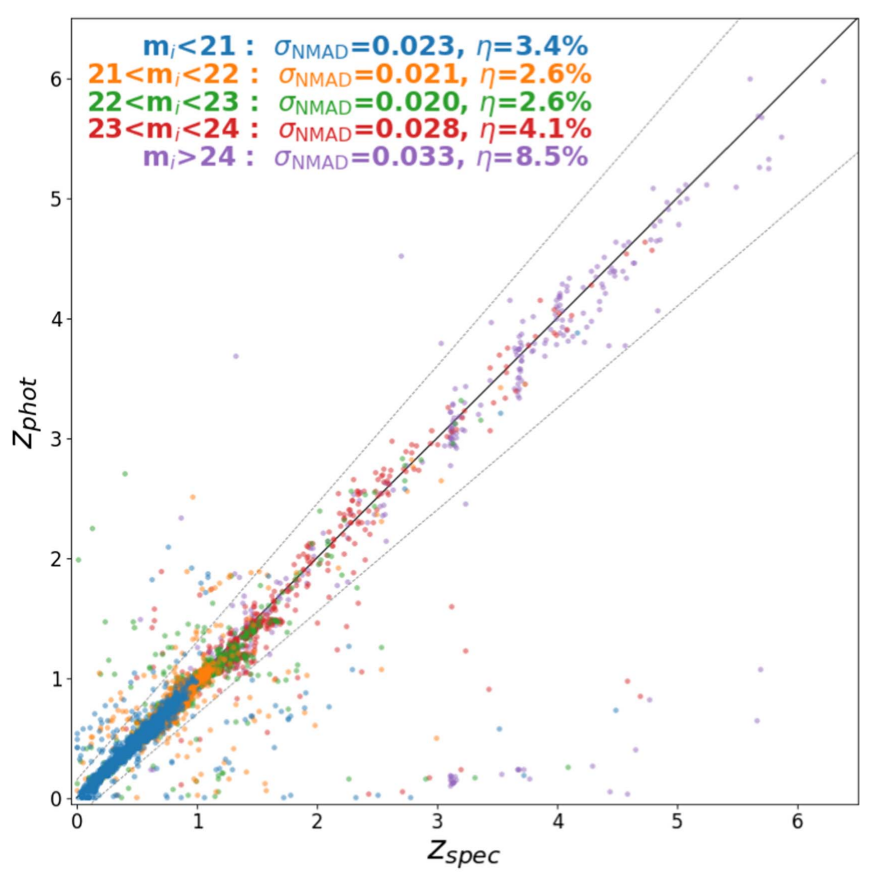

Figure 13. Comparison between the photometric and spectroscopic redshifts as a function of the $i$-band magnitude along with $\sigma_{\mathrm{NMAD}}$ and outlier fraction for each magnitude bin. The dotted lines show the outlier criterion: $z_{\text {phot }}=z_{\text {spec }} \pm 0.15\left(1+z_{\text {spec }}\right)$. Objects below the detection limit in the $B$-band are replaced with their $1 \sigma$ upper limits in the figure.

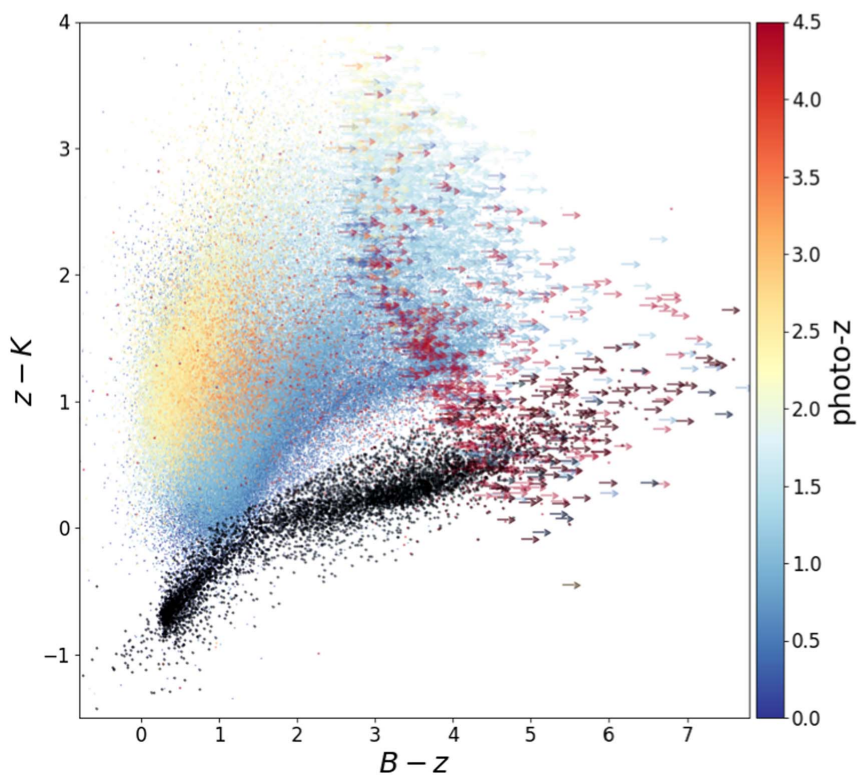

Figure 14. Color-color diagram showing the $B-z$ vs. $z-K_{\mathrm{s}}$ for all sources color-coded according to their photometric redshift. Sources flagged as stars from the star/galaxy classification are shown in black. The upper limits (arrows) are sources that are undetected in the $B$-band and have their magnitudes replaced with the corresponding $1 \sigma$ upper limit.

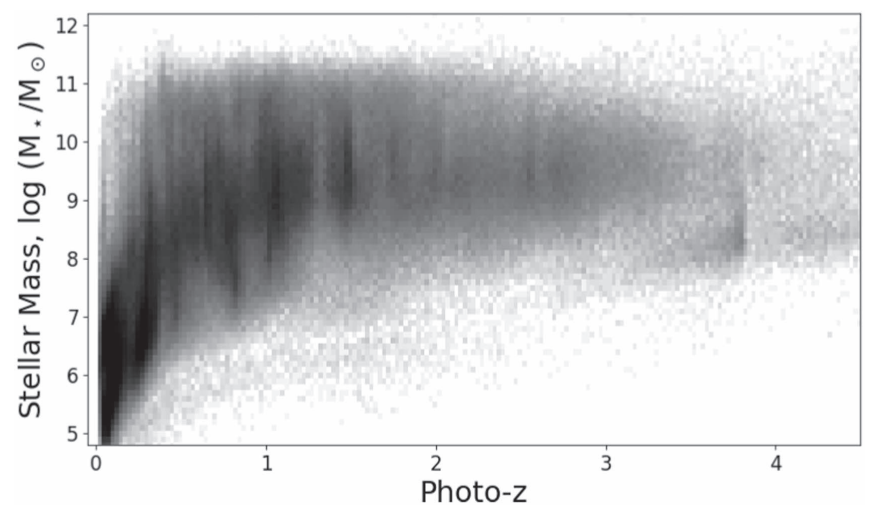

Figure 15. The distribution of stellar masses as estimated from the best-fit template shown as a function of the photometric redshift.

\subsection{Physical Properties}

The physical properties for the objects in our catalog are computed using LEPHARE. Proper estimates of the stellar masses require computation using all the light from the source, hence we use the AUTO magnitudes.

The templates used for measuring the stellar physical properties include Bruzual \& Charlot (2003) models with exponentially declining SFH, with nine timescale values in the range $\tau=0.1-30 \mathrm{Gyr}$ and different metallicities $(Z=0.004$, $Z=0.008$ and $Z=0.02=Z_{\odot}$ ). All models assumed a Chabrier (2003) initial mass function (IMF). We consider 57 ages wellsampled between $0.01 \mathrm{Gyr}$ and $13.5 \mathrm{Gyr}$. As we did when computing the photometric redshifts, emission lines are added to the templates using the empirical relation between the UV luminosity and emission line fluxes, as described in Ilbert et al. (2009). Dust extinction is added to the templates as a free parameter ranging between $0 \leqslant E(B-V) \leqslant 1.2$. The Prevot 
et al. (1984) and Calzetti et al. (2000) extinction laws are considered.

The physical properties are measured by running LePhare with the redshift fixed to the measured photometric redshift (Z_MED from Section 5.1). For sources where $Z_{-}$MED is not estimated, we revert to Z_BEST (redshift from the best-fit template). Estimates for the stellar mass, age, star formation rate, dust attenuation, and best extinction law are reported in the catalog. Figure 15 shows the stellar mass distribution as a function of redshift for the SXDF catalog.

\section{Summary}

We present a photometric catalog for the Subaru/XMMNewton Deep survey field, one of the deep fields with the largest contiguous area covered over a wide wavelength range. We include imaging data in 28 photometric bandpasses spanning from the optical to the mid-infrared. Importantly, we homogenize and assemble all optical and near-infrared data from various instruments and surveys onto a common reference frame to minimize systematic effects. The catalog contains $\sim 1.17$ million objects over an area of $\sim 4.2 \mathrm{deg}^{2}$, with multiwavelength photometry performed using a multi-band detection image, including $\sim 800,000$ objects within the $2.4 \mathrm{deg}^{2}$ HSC-UD area of higher depth and superior wavelength coverage. Exploiting the extensive multi-wavelength coverage, we measure accurate photometric redshifts for all sources. The photometric redshifts are calibrated using $\sim 10,000$ reliable spectroscopic redshifts available from various surveys.

The SPLASH-SXDF catalog is perfectly suited for studying galaxies in the early universe and tracing their evolution through cosmic time. The large area coverage also allows for investigations of the large-scale structure and environmental effects on galaxy evolution, without being significantly affected by cosmic variance.

V.M. would like to thank Micaela Bagley for a helpful discussion regarding the photometric errors. V.M. and C.S. acknowledge the support from Jet Propulsion Laboratory under the grant award \#RSA-1516084. V.M. also acknowledges support from the University of Minnesota Doctoral Dissertation Fellowship 2016-17. W.H.W. acknowledges the support from the Ministry of Science and Technology of Taiwan grant 105-2112-M- 001-029MY3. O.I. acknowledges the funding of the French Agence Nationale de la Recherche for the SAGACE project.

The Cosmic Dawn Center is funded by the Danish National Research Foundation.

This work is based in part on data collected at the Subaru Telescope and retrieved from the HSC data archive system, which is operated by Subaru Telescope and Astronomy Data Center at National Astronomical Observatory of Japan.

This work is based in part on observations made with the Spitzer Space Telescope, which is operated by the Jet Propulsion Laboratory, California Institute of Technology under a contract with NASA. Support for this work was provided by NASA through an award issued by JPL/Caltech.

The Hyper-Suprime-Cam (HSC) collaboration includes the astronomical communities of Japan and Taiwan, and Princeton University. The HSC instrumentation and software were developed by the National Astronomical Observatory of Japan (NAOJ), the Kavli Institute for the Physics and Mathematics of the Universe (Kavli IPMU), the University of Tokyo, the High Energy Accelerator Research Organization (KEK), the Academia Sinica Institute for Astronomy and Astrophysics in Taiwan (ASIAA), and
Princeton University. Funding was contributed by the FIRST program from Japanese Cabinet Office, the Ministry of Education, Culture, Sports, Science and Technology (MEXT), the Japan Society for the Promotion of Science (JSPS), Japan Science and Technology Agency (JST), the Toray Science Foundation, NAOJ, Kavli IPMU, KEK, ASIAA, and Princeton University.

This paper makes use of software developed for the Large Synoptic Survey Telescope. We thank the LSST Project for making their code available as free software at http://dm.lsst.org/.

This work is based in part on observations obtained with MegaPrime and MegaCam, a joint project of CFHT and CEA/ IRFU, at the Canada-France-Hawaii Telescope (CFHT), which is operated by the National Research Council (NRC) of Canada, the Institut National des Science de l'Univers of the Centre National de la Recherche Scientifique (CNRS) of France, and the University of Hawaii. This work is based in part on data products produced at Terapix available at the Canadian Astronomy Data Centre as part of the Canada-France-Hawaii Telescope Legacy Survey, a collaborative project of NRC and CNRS.

This research has made use of the NASA/IPAC Extragalactic Database (NED), which is operated by the Jet Propulsion Laboratory, California Institute of Technology, under contract with the National Aeronautics and Space Administration.

Facilities: Spitzer, Subaru, CFHT, UKIRT, VISTA.

Software: NumPy, SciPy, AstroPy, Matplotlib, PHOTUtILS, LePhare, SEXTRACTOR, SCAMP, SWARP, PSFEx.

\section{Appendix A Catalog Description}

Since the coverage area for individual bands is different, we include a special flag (COVERAGE_FLAG_* ${ }^{*}$ in the final catalog to identify whether an object was covered in a given

Table 5

SEXTRACTOR Parameters Used for Dual Image Mode $\chi^{2}$ Detection and Photometry

\begin{tabular}{ll}
\hline \hline Parameter Name & Value \\
\hline DETECT_TYPE & CCD \\
DETECT_MINAREA & 5 \\
DETECT_MAXAREA & 100000 \\
THRESH_TYPE & ABSOLUTE \\
DETECT_THRESH & 0.51 \\
ANALYSIS_THRESH & 0.5 \\
FILTER & $\mathrm{Y}$ \\
FILTER_NAME & Gauss_3.0_5 $\times 5$. conv \\
DEBLEND_NTHRESH & 32 \\
DEBLEND_MINCONT & 0.00001 \\
CLEAN & $\mathrm{Y}$ \\
CLEAN_PARAM & 1.0 \\
MASK_TYPE & $\mathrm{CORRECT}$ \\
WEIGHT_GAIN & $\mathrm{N}$ \\
RESCALE_WEIGHTS & $\mathrm{N}$ \\
PHOT_APERTURES & $6.67,13.33,20.00,26.67,33.33$ \\
PHOT_AUTOPARAMS & $2.5,3.5$ \\
PHOT_AUTOAPERS & 10,10 \\
PHOT_FLUXFRAC & $0.2,0.5,0.8$ \\
MAG_ZEROPOINT & 23.93 \\
GAIN & 0.0 \\
GAIN_KEY & DUMMY \\
BACK_SIZE & 128 \\
BACK_FILTERSIZE & 3 \\
BACKPHOTO_TYPE & LOCAL \\
BACKPHOTO_THICK & 30 \\
\hline
\end{tabular}


Table 6

Correction Factors for SEXTRACTOR Photometric Errors

\begin{tabular}{|c|c|c|c|c|c|c|}
\hline \multirow{2}{*}{$\begin{array}{l}\text { Instrument/ } \\
\text { Survey }\end{array}$} & \multirow{2}{*}{ Filters } & \multicolumn{5}{|c|}{ Aperture Size } \\
\hline & & $1^{\prime \prime}$ & $2^{\prime \prime}$ & $3^{\prime \prime}$ & $4^{\prime \prime}$ & $5^{\prime \prime}$ \\
\hline \multirow[t]{5}{*}{$\overline{\mathrm{HSC}}$} & $g$ & 1.26 & 1.70 & 2.18 & 2.70 & 3.16 \\
\hline & $r$ & 1.26 & 1.72 & 2.20 & 2.69 & 3.15 \\
\hline & $i$ & 1.29 & 1.82 & 2.31 & 2.87 & 3.38 \\
\hline & $z$ & 1.19 & 1.57 & 1.96 & 2.36 & 2.76 \\
\hline & $y$ & 1.19 & 1.60 & 2.02 & 2.47 & 2.91 \\
\hline \multirow[t]{5}{*}{ SupCam } & $B$ & $2.11,2.30$ & $3.29,3.69$ & $4.41,5.08$ & $5.47,6.36$ & $6.40,7.45$ \\
\hline & $V$ & $2.35,2.64$ & $3.69,4.21$ & $4.92,5.63$ & $5.99,6.81$ & $6.84,7.72$ \\
\hline & $R_{c}$ & $2.19,2.44$ & $3.28,3.78$ & $4.27,4.98$ & $5.19,5.98$ & $5.90,6.78$ \\
\hline & $i^{\prime}$ & $2.64,2.93$ & $4.33,5.09$ & $5.83,7.01$ & $7.15,8.68$ & $8.22,10.08$ \\
\hline & $z^{\prime}$ & $2.49,2.61$ & $3.46,3.85$ & $4.21,4.84$ & $4.97,5.76$ & $5.61,6.55$ \\
\hline \multirow[t]{3}{*}{ UDS } & $J$ & 0.96 & 1.07 & 1.12 & 1.17 & 1.21 \\
\hline & $H$ & 0.93 & 1.01 & 1.03 & 1.05 & 1.07 \\
\hline & $K$ & 0.96 & 1.07 & 1.12 & 1.18 & 1.23 \\
\hline \multirow[t]{5}{*}{ VIDEO } & $Z$ & 2.86 & 4.32 & 5.68 & 7.09 & 8.51 \\
\hline & $Y$ & 2.45 & 3.44 & 4.39 & 5.36 & 6.32 \\
\hline & $J$ & $2.21,2.26$ & $2.72,2.99$ & $3.14,3.60$ & $3.59,4.26$ & $4.03,4.94$ \\
\hline & $H$ & 2.30 & 3.03 & 3.63 & 4.20 & 4.78 \\
\hline & $K_{s}$ & 2.21 & 2.69 & 3.10 & 3.53 & 3.94 \\
\hline MUSUBI & $u$ & $0.18,0.21,0.19$ & $0.24,0.31,0.26$ & $0.30,0.39,0.32$ & $0.35,0.48,0.39$ & $0.40,0.56,0.45$ \\
\hline \multirow[t]{5}{*}{ CFHTLS } & $u$ & 0.61 & 0.68 & 0.73 & 0.80 & 0.86 \\
\hline & $g$ & 1.05 & 1.21 & 1.34 & 1.47 & 1.60 \\
\hline & $r$ & 0.85 & 0.98 & 1.08 & 1.20 & 1.31 \\
\hline & $i$ & 0.74 & 0.92 & 1.10 & 1.27 & 1.43 \\
\hline & $z$ & 0.97 & 1.58 & 2.14 & 2.66 & 3.11 \\
\hline
\end{tabular}

band. For the optical and NIR filters, this flag identifies whether imaging data is available at the source position in the given band. In the case of the HSC filters, an object may also not be covered due to the star mask, which is also included in the COVERAGE_FLAG.

Table 5 lists the parameters used for the extraction of photometry using SEXTRACTOR in dual image mode with the $\chi^{2}$ detection image. Table 7 describes all the columns available in the catalog. A compressed version of the catalog (in binary FITS table format) is available for download here: https://z. umn.edu/SXDF.

\section{Appendix B Correcting Photometric Errors}

In order to properly account for the photometric errors, we measure the variation in the sky noise and use it to correct the photometric errors computed from the weight maps by SEXTRACTOR. The motivation for this comes from the fact that the sky variation can properly account for undetected faint objects and correlated noise that the weight maps cannot. Moreover, no weight maps were available for the Suprime-Cam data and we had to use a smoothed background rms map instead. With the proposed treatment, the photometric errors would be made consistent across all bands.

For each source, we measure the average value of the weight map at its location in each band. Using the aperture size and weight value (proxy for image depth), we can compute the expected photometric error based on the results of the empty sky aperture analysis (Section 4.3.2). We can then compute a median correction factor to adjust the SEXTRACTOR-measured photometric errors to the value determined from the sky noise. We only consider sources in the mid-50\% of the magnitude distribution, avoiding potential biases from the faintest and brightest sources. This correction is expected to scale with the aperture size. Additionally, we find this factor to differ for regions of the mosaic with different depths. In order to account for both these effects, we compute a separate aperture-sizedependent correction factor at different depths. This only affects the Suprime-Cam $B V R_{c} i^{\prime} z^{\prime}$ images that are divided into two subregions and the MUSUBI- $u$ mosaic that is divided into three subregions. Table 6 lists the correction factors for each band in the $1^{\prime \prime}, 2^{\prime \prime}, 3^{\prime \prime}, 4^{\prime \prime}$, and $5^{\prime \prime}$ apertures. 
Table 7

Column Descriptions for the SPLASH-SXDS Catalog v1.4

\begin{tabular}{|c|c|c|}
\hline Column No. & Column Title & Description \\
\hline \multicolumn{3}{|r|}{ General Information } \\
\hline 1 & ID & Source Identification number \\
\hline 2 & RA & Right Ascension [deg] \\
\hline 3 & $\mathrm{DEC}$ & Declination $[\mathrm{deg}]$ \\
\hline 4 & A & Semimajor axis [deg] \\
\hline 5 & $\mathrm{~B}$ & Semiminor axis $[\mathrm{deg}]$ \\
\hline 6 & THETA & Position Angle [deg] \\
\hline 7 & X_IMAGE & Object position along $\mathrm{x}[\mathrm{px}]$ \\
\hline 8 & Y_IMAGE & Object position along y $[\mathrm{px}]$ \\
\hline 9 & A_IMAGE & Semimajor axis $[\mathrm{px}]$ \\
\hline 10 & B_IMAGE & Semiminor axis $[\mathrm{px}]$ \\
\hline 11 & THETA_IMAGE & Position Angle [deg] \\
\hline 12 & KRON_RADIUS & Kron apertures in units of A or B \\
\hline 13 & PETRO_RADIUS & Petrosian apertures in units of $\mathrm{A}$ or $\mathrm{B}$ \\
\hline 14 & ISOAREAF_IMAGE & Isophotal area (filtered) above Detection threshold [pixel $\left.{ }^{* *} 2\right]$ \\
\hline 15 & ELONGATION & A_IMAGE/B_IMAGE \\
\hline 16 & ELLIPTICITY & 1-B_IMAGE/A_IMAGE \\
\hline 17 & GAL_EXT_EBV & Galactic Extinction, E(B - V) \\
\hline 18 & OFFSET_FLUX & Offset (multiplicative) between AUTO and APER fluxes for $1^{\prime \prime}, 2^{\prime \prime}, 3^{\prime \prime}, 4^{\prime \prime}, 5^{\prime \prime}$ apertures \\
\hline 19 & OFFSET_MAG & Offset (linear) between AUTO and APER magnitudes for $1^{\prime \prime}, 2^{\prime \prime}, 3^{\prime \prime}, 4^{\prime \prime}, 5^{\prime \prime}$ apertures \\
\hline 20 & ZSPEC & Spectroscopic redshift, if available (Proprietary spec-z's are flagged with -1 ) \\
\hline 21 & ZSPEC_REF & Reference for the spectroscopic redshift \\
\hline 22 & ZPHOT & Best redshift for the source ${ }^{\mathrm{a}}$ \\
\hline 23 & STAR_FLAG & Star/galaxy classification flag : $1=$ star, $0=$ galaxy, $-99=$ no classification available \\
\hline \multicolumn{3}{|r|}{ Photometry $^{\mathrm{b}}$} \\
\hline $24-383$ & MAG_AUTO_* & Kron-like elliptical aperture magnitude $[\mathrm{AB}]$ \\
\hline & MAGERR_AUTO_* & Error for AUTO magnitude $[\mathrm{AB}]$ \\
\hline & FLUX_AUTO_* & Flux within a Kron-like elliptical aperture $[\mu \mathrm{Jy}]$ \\
\hline & FLUXERR_AUTO_* & Error on AUTO flux $[\mu \mathrm{Jy}]$ \\
\hline & MAG_ISO_* & Isophotal magnitude [AB] \\
\hline & MAGERR_ISO_* & Error for isophotal magnitude $[\mathrm{AB}]$ \\
\hline & FLUX_ISO_* & Isophotal flux $[\mu \mathrm{Jy}]$ \\
\hline & FLUXERR_ISO_* & Error on Isophotal flux $[\mu \mathrm{Jy}]$ \\
\hline & MAG_APER_* & Fixed circular aperture magnitudes for $1^{\prime \prime}, 2^{\prime \prime}, 3^{\prime \prime}, 4^{\prime \prime}, 5^{\prime \prime}$ apertures $[\mathrm{AB}]^{\mathrm{c}}$ \\
\hline & MAGERR_APER_* & Error on APER magnitudes $[\mathrm{AB}]^{\mathrm{c}}$ \\
\hline & FLUX_APER_* & Fluxes within $1^{\prime \prime}, 2^{\prime \prime}, 3^{\prime \prime}, 4^{\prime \prime}, 5^{\prime \prime}$ circular apertures $[\mu \mathrm{Jy}]$ \\
\hline & FLUXERR_APER_* & Errors on APER fluxes $[\mu \mathrm{Jy}]$ \\
\hline & FLUX_RADIUS_* & Fraction of light radii for $20 \%, 50 \%, 80 \%$ [px] \\
\hline & SE_FLAGS_* & SExtractor extraction flags \\
\hline & COVERAGE_FLAG_* & Coverage flag: $1=$ covered in filter, $0=$ not covered \\
\hline \multicolumn{3}{|r|}{ IRAC Photometry } \\
\hline $384-407$ & MAG_TOT_irac_* & Total IRAC magnitude $[\mathrm{AB}]^{\mathrm{c}}$ \\
\hline & MAGERR_TOT_irac_* & Error for total IRAC magnitude $[\mathrm{AB}]^{\mathrm{c}}$ \\
\hline & FLUX_TOT_irac_* & Total IRAC Flux $[\mu \mathrm{Jy}]$ \\
\hline & FLUXERR_TOT_irac_* & Error on total IRAC flux $[\mu \mathrm{Jy}]$ \\
\hline & SE_FLAGS_irac_* & Source extraction flags ${ }^{\mathrm{d}}$ for IRACLEAN \\
\hline & COVERAGE_FLAG_irac_* & Coverage flag: $1=$ covered in filter, $0=$ not covered \\
\hline \multicolumn{3}{|r|}{ Radio catalog informations (Simpson et al. 2006) } \\
\hline 408 & 1_4GHz_ID & Source ID in the radio catalog \\
\hline 409 & 1_4GHz_RA & Right Ascension of the radio source [deg] \\
\hline 410 & 1_4GHz_DEC & Declination of the radio source [deg] \\
\hline 411 & 1_4GHz_FLUX & $1.4 \mathrm{GHz}$ total Flux $[\mu \mathrm{Jy}]$ \\
\hline 412 & 1_4GHz_FLUXERR & $1.4 \mathrm{GHz}$ total Flux error $[\mu \mathrm{Jy}]$ \\
\hline 413 & 1_4GHz_Rel & Reliability of the optical identification \\
\hline 414 & 1_4GHz_n_Rel & Note when no reliability value: $\mathrm{A}=$ reliable, $\mathrm{B}=$ probable, $\mathrm{C}=$ plausible, $-=$ certain identification \\
\hline \multicolumn{3}{|r|}{ X-ray catalog informations (Akiyama et al. 2015) } \\
\hline 415 & Xray_ID & Source ID in the X-ray catalog \\
\hline 416 & Xray_RA & Right Ascension of the X-ray source [deg] \\
\hline
\end{tabular}


Table 7

(Continued)

\begin{tabular}{|c|c|c|}
\hline Column No. & Column Title & Description \\
\hline 418 & Xray_zUSE & Redshift used for NH, LHX estimation \\
\hline 419 & Xray_HR2 & Hardness ratio \\
\hline 421 & Xray_logLHX & Log of best-estimated X-ray luminosity $\left[10^{-7} \mathrm{~W}\right]$ \\
\hline \multicolumn{3}{|r|}{ LEPHARE run for Photo-z measurement } \\
\hline 424 & Z_MED_U68 & Upper limit of $68 \%$ CI for the $Z_{-}$MED \\
\hline 425 & Z_BEST & Best estimate of photometric redshift \\
\hline 426 & Z_BEST_L68 & Lower limit of $68 \% \mathrm{CI}$ for the $\mathrm{Z} \_\mathrm{BEST}$ \\
\hline 427 & Z_BEST_U68 & Upper limit of $68 \%$ CI for the Z_BEST \\
\hline 428 & CHI_BEST & Reduced $\chi^{2}$ value for best-fit \\
\hline 429 & PDZ_BEST & Probability dist. between $z_{\text {best }} \pm 0.1(1+z)$ \\
\hline 434 & CHI_STAR & Reduced $\chi^{2}$ value for the best solution with the STAR library \\
\hline 435 & NBAND_Z & No. of bands used for the photometric redshift fit \\
\hline \multicolumn{3}{|r|}{ LEPHARE run for physical properties } \\
\hline 436 & EBV_BEST & $\mathrm{E}(\mathrm{B}-\mathrm{V})$ \\
\hline 437 & EXTLAW_BEST & Extinction law used: $1=$ Prevot et al. (1984); $2=$ Calzetti et al. (2000) \\
\hline 438 & MASS_BEST & Mass [solar masses] \\
\hline 439 & AGE_BEST & Age $[\mathrm{yr}]$ \\
\hline 440 & SFR_BEST & Star formation rate [solar masses per year] \\
\hline 441 & SSFR_BEST & Specific SFR $[1 / y r]$ \\
\hline 442 & CHI_PHYS & Reduced $\chi^{2}$ value for the best-fit physical template \\
\hline 443 & LUM_NUV_BEST & NUV luminosity from best-fit model [solar luminosities] \\
\hline
\end{tabular}

Notes.

a This is set to the estimate from the median of the probability distribution function (Z_MED) for all objects except those flagged as stars (STAR_FLAG), which are set to 0 . When Z_MED is unavailable, Z_BEST is used instead.

${ }^{\mathrm{b}}$ Each filter has its own entry. The columns are suffixed by $<$ instr_name $>$ _ $<$ filt_name $>$.

${ }^{\mathrm{c}}$ If the magnitude error is set to -1 , the magnitude represents the $1 \sigma$ upper limit.

${ }^{\mathrm{d}} 1=$ no photometry because the object was $<1 \mathrm{px}$ at IRAC resolution and adjacent to a bright object; 2 = no photometry because there is no IRAC coverage at the object position.

${ }^{\mathrm{e}}$ The absolute magnitude for all 28 photometric bands estimated from the best-fit template.

\section{ORCID iDs}

Vihang Mehta (1) https://orcid.org/0000-0001-7166-6035 Claudia Scarlata 낭 https://orcid.org/0000-0002-9136-8876 Iary Davidzon (1) https://orcid.org/0000-0002-2951-7519 Andreas Faisst @ https://orcid.org/0000-0002-9382-9832 Bau Ching Hsieh (1) https://orcid.org/0000-0001-5615-4904 Olivier Ilbert iㅛ https://orcid.org/0000-0002-7303-4397 John Silverman (1) https://orcid.org/0000-0002-0000-6977 Michael A. Strauss (i) https://orcid.org/0000-0002-0106-7755 Masayuki Tanaka (i) https://orcid.org/0000-0002-5011-5178 Sébastien Foucaud (1) https://orcid.org/0000-0001-5603-6262 Shoubaneh Hemmati 10 https://orcid.org/0000-00032226-5395

Daniel Masters ํㅏ https://orcid.org/0000-0001-5382-6138
Henry Joy McCracken (1) https://orcid.org/0000-00029489-7765

Masami Ouchi 나 https://orcid.org/0000-0002-1049-6658

Wei-Hao Wang @ https://orcid.org/0000-0003-2588-1265

\section{References}

Aihara, H., Allende Prieto, C., An, D., et al. 2011, ApJS, 193, 29 Aihara, H., Arimoto, N., Armstrong, R., et al. 2017a, arXiv:1704.05858 Aihara, H., Armstrong, R., Bickerton, S., et al. 2017b, arXiv:1702.08449 Akiyama, M., Ueda, Y., Watson, M. G., et al. 2015, PASJ, 67, 82 Arnouts, S., Cristiani, S., Moscardini, L., et al. 1999, MNRAS, 310, 540 Ashby, M. L. N., Willner, S. P., Fazio, G. G., et al. 2013, ApJ, 769, 80 Ashby, M. L. N., Willner, S. P., Fazio, G. G., et al. 2015, ApJS, 218, 33 Baraffe, I., Homeier, D., Allard, F., \& Chabrier, G. 2015, A\&A, 577, A42 Bell, E. F., van der Wel, A., Papovich, C., et al. 2012, ApJ, 753, 167 
Bertin, E. 2011, in ASP Conf. Ser. 442, Astronomical Data Analysis Software and Systems XX, ed. I. N. Evans et al. (San Francisco, CA: ASP), 435

Bertin, E., \& Arnouts, S. 1996, A\&AS, 117, 393

Bertin, E., Mellier, Y., Radovich, M., et al. 2002, in ASP Conf. Ser 281, Astronomical Data Analysis Software and Systems XI, ed. D. A. Bohlender, D. Durand, \& T. H. Handley (San Francisco, CA: ASP), 228

Bixler, J. V., Bowyer, S., \& Laget, M. 1991, A\&A, 250, 370

Bosch, J., Armstrong, R., Bickerton, S., et al. 2017, arXiv:1705.06766

Bradley, L., Sipocz, B., Robitaille, T., et al. 2016, Photutils: Photometry tools, Astrophysics Source Code Library, ascl:1609.011

Bradshaw, E. J., Almaini, O., Hartley, W. G., et al. 2013, MNRAS, 433, 194

Bruzual, G., \& Charlot, S. 2003, MNRAS, 344, 1000

Calzetti, D., Armus, L., Bohlin, R. C., et al. 2000, ApJ, 533, 682

Caputi, K. I., Cirasuolo, M., Dunlop, J. S., et al. 2011, MNRAS, 413, 162

Cardelli, J. A., Clayton, G. C., \& Mathis, J. S. 1989, ApJ, 345, 245

Chabrier, G. 2003, PASP, 115, 763

Chabrier, G., Baraffe, I., Allard, F., \& Hauschildt, P. 2000, ApJ, 542, 464

Curtis-Lake, E., McLure, R. J., Pearce, H. J., et al. 2012, MNRAS, 422, 1425

Darvish, B., Mobasher, B., Martin, D. C., et al. 2017, ApJ, 837, 16

Darvish, B., Mobasher, B., Sobral, D., et al. 2016, ApJ, 825, 113

Davidzon, I., Ilbert, O., Laigle, C., et al. 2017, A\&A, 605, A70

Finoguenov, A., Watson, M. G., Tanaka, M., et al. 2010, MNRAS, 403, 2063

Fitzpatrick, E. L. 1986, AJ, 92, 1068

Franx, M., van Dokkum, P. G., Förster Schreiber, N. M., et al. 2008, ApJ, 688, 770

Furusawa, H., Kosugi, G., Akiyama, M., et al. 2008, ApJS, 176, 1

Garilli, B., Guzzo, L., Scodeggio, M., et al. 2014, A\&A, 562, A23

Geach, J. E., Simpson, C., Rawlings, S., Read, A. M., \& Watson, M. 2007, MNRAS, 381, 1369

Guzzo, L., Scodeggio, M., Garilli, B., et al. 2014, A\&A, 566, A108

Hildebrandt, H., Erben, T., Kuijken, K., et al. 2012, MNRAS, 421, 2355

Hoaglin, D. C., Mosteller, F., \& Tukey, J. W. 1983, Understanding Robust and Exploratory Data Analysis (New York: Wiley)

Hsieh, B.-C., Wang, W.-H., Hsieh, C.-C., et al. 2012, ApJS, 203, 23

Ilbert, O., Arnouts, S., McCracken, H. J., et al. 2006, A\&A, 457, 841

Ilbert, O., Capak, P., Salvato, M., et al. 2009, ApJ, 690, 1236

Ilbert, O., McCracken, H. J., Le Fèvre, O., et al. 2013, A\&A, 556, A55

Ilbert, O., Salvato, M., Le Floc'h, E., et al. 2010, ApJ, 709, 644

Jarvis, M. J., Bonfield, D. G., Bruce, V. A., et al. 2013, MNRAS, 428, 1281

Kauffmann, G., White, S. D. M., Heckman, T. M., et al. 2004, MNRAS, 353, 713

Kron, R. G. 1980, ApJS, 43, 305

Laigle, C., McCracken, H. J., Ilbert, O., et al. 2016, ApJS, 224, 24

Laigle, C., Pichon, C., Arnouts, S., et al. 2017, arXiv:1702.08810

Lawrence, A., Warren, S. J., Almaini, O., et al. 2007, MNRAS, 379, 1599

Lonsdale, C. J., Smith, H. E., Rowan-Robinson, M., et al. 2003, PASP, 115, 897

Marchesini, D., van Dokkum, P. G., Förster Schreiber, N. M., et al. 2009, ApJ, 701,1765

Massarotti, M., Iovino, A., Buzzoni, A., \& Valls-Gabaud, D. 2001, A\&A, 380,425
Massey, R., \& Refregier, A. 2005, MNRAS, 363, 197

Masters, D. C., Stern, D. K., Cohen, J. G., et al. 2017, ApJ, 841, 111

Matsuoka, Y., Onoue, M., Kashikawa, N., et al. 2016, ApJ, 828, 26

Mauduit, J.-C., Lacy, M., Farrah, D., et al. 2012, PASP, 124, 714

McLure, R. J., Pearce, H. J., Dunlop, J. S., et al. 2013, MNRAS, 428, 1088

Miyazaki, S., Komiyama, Y., Kawanomoto, S., et al. 2018, PASJ, 70, 1

Miyazaki, S., Komiyama, Y., Nakaya, H., et al. 2012, Proc. SPIE, 8446, $84460 Z$

Moffat, A. F. J. 1969, A\&A, 3, 455

Momcheva, I. G., Brammer, G. B., van Dokkum, P. G., et al. 2016, ApJS, 225,27

Moutard, T., Arnouts, S., Ilbert, O., et al. 2016, A\&A, 590, A102

Muzzin, A., Marchesini, D., Stefanon, M., et al. 2013, ApJ, 777, 18

Oke, J. B., \& Gunn, J. E. 1983, ApJ, 266, 713

Ono, Y., Ouchi, M., Harikane, Y., et al. 2017, arXiv:1704.06004

Onodera, M., Renzini, A., Carollo, M., et al. 2012, ApJ, 755, 26

Ouchi, M., Shimasaku, K., Akiyama, M., et al. 2005, ApJL, 620, L1

Ouchi, M., Shimasaku, K., Akiyama, M., et al. 2008, ApJS, 176, 301

Ouchi, M., Shimasaku, K., Furusawa, H., et al. 2010, ApJ, 723, 869

Pâris, I., Petitjean, P., Ross, N. P., et al. 2017, A\&A, 597, A79

Peng, Y.-j., Lilly, S. J., Kovač, K., et al. 2010, ApJ, 721, 193

Pickles, A. J. 1998, PASP, 110, 863

Polletta, M., Tajer, M., Maraschi, L., et al. 2007, ApJ, 663, 81

Prevot, M. L., Lequeux, J., Prevot, L., Maurice, E., \& Rocca-Volmerange, B. 1984, A\&A, 132, 389

Saito, T., Shimasaku, K., Okamura, S., et al. 2008, ApJ, 675, 1076

Santini, P., Ferguson, H. C., Fontana, A., et al. 2015, ApJ, 801, 97

Sawicki, M. 2012, PASP, 124, 1208

Schlegel, D. J., Finkbeiner, D. P., \& Davis, M. 1998, ApJ, 500, 525

Scodeggio, M., Guzzo, L., Garilli, B., et al. 2016, arXiv:1611.07048

Scoville, N., Arnouts, S., Aussel, H., et al. 2013, ApJS, 206, 3

Scoville, N., Aussel, H., Brusa, M., et al. 2007, ApJS, 172, 1

Shibuya, T., Ouchi, M., Harikane, Y., et al. 2017, arXiv:1705.00733

Simpson, C., Martínez-Sansigre, A., Rawlings, S., et al. 2006, MNRAS, 372,741

Soto, E., de Mello, D. F., Rafelski, M., et al. 2017, ApJ, 837, 6

Speagle, J. S., Steinhardt, C. L., Capak, P. L., \& Silverman, J. D. 2014, ApJS, 214,15

Szalay, A. S., Connolly, A. J., \& Szokoly, G. P. 1999, AJ, 117, 68

Ueda, Y., Watson, M. G., Stewart, I. M., et al. 2008, ApJS, 179, 124

van Breukelen, C., Cotter, G., Rawlings, S., et al. 2007, MNRAS, 382, 971

van der Wel, A., Bell, E. F., Häussler, B., et al. 2012, ApJS, 203, 24

Wang, F., Wu, X.-B., Fan, X., et al. 2016, ApJ, 819, 24

Whitaker, K. E., Franx, M., Leja, J., et al. 2014, ApJ, 795, 104

Wilkins, S. M., Stanway, E. R., \& Bremer, M. N. 2014, MNRAS, 439, 1038

Wuyts, S., Förster Schreiber, N. M., Genzel, R., et al. 2012, ApJ, 753, 114

Yamada, T., Kodama, T., Akiyama, M., et al. 2005, ApJ, 634, 861 